\title{
6-m telescope spectroscopic observations of the bubble complex in NGC 6946
}

\author{
Yu. N. Efremov ${ }^{1}$, S. A. Pustilnik ${ }^{2,3}$, A. Y. Kniazev ${ }^{2,3,4}$, B. G. Elmegreen ${ }^{5}$, S. S. Larsen ${ }^{6}$, E. J. Alfaro ${ }^{7}$, \\ P. W. Hodge ${ }^{8}$, A. G. Pramsky ${ }^{2,3}$, and T. Richtler ${ }^{9}$ \\ 1 Sternberg Astronomical Institute of Moscow State University, Universitetsky Prospect, 13, Moscow, \\ 119899, Russia \\ 2 Special Astrophysical Observatory of RAS, Nizhnij Arkhyz, Karachai-Circassia, 369167, Russia \\ 3 Isaac Newton Institute of Chile, SAO Branch, Russia \\ 4 Max Planck Institut für Astronomie, Königstuhl 17, 69117, Heidelberg, Germany \\ 5 IBM Research Division, T.J. Watson Research Center, PO Box 218, Yorktown Heights, NY 10598, USA \\ 6 UC Observatories / Lick Observatory, University of California, Santa Cruz, CA 95064, USA \\ 7 Instituto de Astrofisica de Andalucia, 18008 Granada, Spain \\ 8 University of Washington, Seattle, USA \\ 9 Grupo de Astronomía, Departamento de Física, Casilla 160-C, Universidad de Concepción, Concepción, Chile
}

Received 9 September 2001 / Accepted 16 May 2002

\begin{abstract}
We describe the results of a long-slit spectroscopic study of an unusual star complex in the nearby spiral galaxy NGC 6946 using the SAO $6 \mathrm{~m}$ telescope and the Keck $10 \mathrm{~m}$ telescope. The complex resembles a circular bubble 600 pc in diameter with a young super star cluster (SSC) near the center. The kinematics of ionized gas is studied through $\mathrm{H} \alpha$ emission with several slit positions. Position-velocity diagrams show two distinct features with high speed motions. One is an irregularly shaped region to the east of the SSC, $270 \mathrm{pc}$ in size, in which most of the $\mathrm{H} \alpha$ emission is blue shifted by $120 \mathrm{~km} \mathrm{~s}^{-1}$, and another is a $350 \mathrm{pc}$ shell centered on the SSC with positive and negative velocity shifts of $60 \mathrm{~km} \mathrm{~s}^{-1}$. Balmer and He I absorption lines in the SSC give an age of 12-13 Myr, which is consistent with the photometric age but significantly older than the kinematic ages of the high speed regions. The energetics of the SSC and its interaction with the environment are considered. The expansion energies exceed $10^{52} \mathrm{ergs}$, but the power outputs from winds and supernova in the SSC are large enough to account for this. The intensities of Balmer, [N II], and [S II] emission lines within and around the complex indicate that shock excitation makes a significant contribution to the emission from the most energetic region.
\end{abstract}

Key words. galaxies: ISM - galaxies: globular clusters: general - galaxies: formation - galaxies: individual: NGC 6946 - ISM: bubbles

\section{Introduction}

Star complexes combine young clusters and associations along with slightly older stars, like red supergiants and Cepheids, into vast groups $0.5-1.0 \mathrm{kpc}$ in size (Efremov 1995). Most form by common processes related to spiral arm compression, gaseous self-gravity, and interstellar turbulence, and represent the largest scale in the hierarchy of structures related to star formation (Elmegreen et al. 2000b). Others look very unusual with giant stellar arcs inside or at their borders making a near perfect segment of a circle. These arc-like complexes are very rare (only a dozen are known), suggesting that some special process or event was involved with their formation (Efremov 2001, 2002).

Send offprint requests to: Yu. N. Efremov, e-mail: efremov@sai.msu.ru
The first arc-shaped complex in a galaxy was noted by Westerlund \& Mathewson (1966) in the northeast part of the Large Magellanic Cloud (LMC), inside the HI superbubble that is now known as LMC4 from the survey by Meaburn (1980). Hodge (1967) noted this arc also and found two others in the same region plus a similar feature in the spiral galaxy NGC 6946.

The complex in NGC 6946 was recently rediscovered by Larsen \& Richtler (1999). It has a massive young super star cluster (SSC) (Larsen \& Richtler 1999; Efremov 1999) that is $\sim 15 \mathrm{Myr}$ old, and numerous other smaller clusters with about the same age (Elmegreen et al. 2000a). The western edge is a 130 degree-long arc with a radius of $\sim 300$ pc. There is no obvious pattern of stellar age with radius inside the bubble. Models of the color magnitude diagram observed with HST suggest that the SSC was triggered in a centralized molecular cloud by compression from a surrounding OB association; evolved remnants 


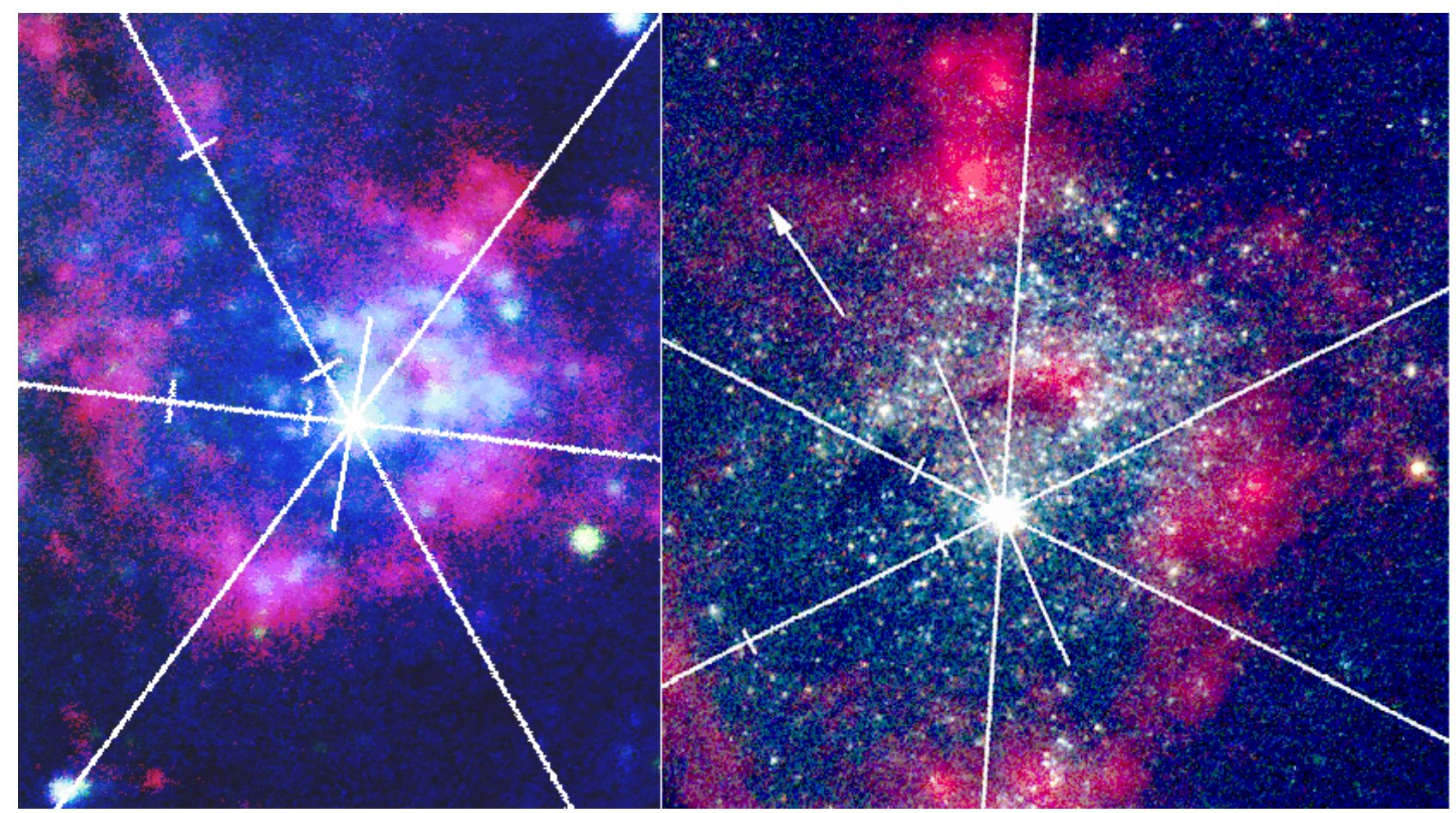

Fig. 1. The positions of the long slits from the 6-m telescope and the shorter slit from Keck I are superposed on a combined image using $\mathrm{H} \alpha, B, V$, and $R$ from the Nordic Optical Telescope (left) and a combined image with the same H $\alpha$ from NOT and $B, V$, and $R$ from HST (right). For the left-hand image, North is up, East is to the left, and the full extent East-West is $43^{\prime \prime}$. For for the right-hand image, North is indicated by the arrow and the full extend left-right is $20.9^{\prime \prime}$. Tic marks indicate ranges of positions where the centroid velocity of the $\mathrm{H} \alpha$ line shifts to large negative values.

indicate that this association formed 20 to $30 \mathrm{Myr}$ ago and contained 1000 OB-type stars (Larsen et al. 2002). Pieces of the cloud may be visible today as two $10^{5} M_{\odot}$ dust arcs $150 \mathrm{pc}$ to the north and northwest of the SSC (Elmegreen et al. 2000a). The HST photometry was also combined with a velocity dispersion profile obtained at the Keck I telescope to give an SSC mass of $\sim 10^{6} M_{\odot}$ and a central density of $\sim 10^{4} M_{\odot} \mathrm{pc}^{-3}$ (Larsen et al. 2001).

The origin and evolution of the complex with its peculiar circular shape and massive cluster are unclear. More data on the kinematics of the region are needed. For this reason, we observed the complex with three spectroscopic slits using the $6 \mathrm{~m}$ telescope of the Special Astrophysical Observatory of the Russian Academy of Sciences. We also studied another slit spectrum from the same Keck I observations as those used by Larsen et al. (2001).

Here we discuss the gas kinematics that is evident from this spectroscopy, and we derive several cluster ages from the Balmer lines.

\section{Observations and data reduction}

\subsection{Long-slit spectroscopy with the $6 \mathrm{~m}$ telescope}

Figure 1 shows two color images of the star complex. The image on the left combines $\mathrm{H} \alpha, B, V$, and $R$ from the Nordic Optical Telescope and the image on the right combines the same $\mathrm{H} \alpha$ exposure with $B, V$, and $R$ from HST. Four slit positions used for this study are superposed.
Tic marks on the slits indicate the ranges of positions where fast $\mathrm{H} \alpha$ emission is found (see below).

The observations for three of the slits were done on the $6 \mathrm{~m}$ telescope of the Special Astrophysical Observatory of the Russian Academy of Sciences (SAO RAS) during 2 nights in July 2000 (see Table 1 for details). The Long-Slit spectrograph (Afanasiev et al. 1995) at the telescope prime focus was equipped with a Photometrics CCD-detector with $1024 \times 1024$ pixels and $24 \times 24 \mu \mathrm{m}$ pixel size. The slit length is $130^{\prime \prime}$. The spectra used a grating with 1302 grooves/mm and a dispersion of $1.2 \AA /$ pixel, giving a spectral resolution of $3.2 \AA$ at $\mathrm{H} \alpha$. The slit positions (see Fig. 1) were chosen to cross the SSC and various characteristic features of this complex, including bright $\mathrm{H}$ II regions and faint clusters near the SSC. The slit with $\mathrm{PA}=83^{\circ}$ points between the direction to the galaxy center $\left(\mathrm{PA}=70^{\circ}\right)$ and the tangent direction to the local spiral arm $\left(\mathrm{PA} \sim 93^{\circ}\right)$. The slit position with $\mathrm{PA}=29^{\circ}$ runs across the arm.

The wavelength ranges for the spectra are given in Table 1. A slit width of $2^{\prime \prime}$ was used in all cases. The scale along the slit is $0.39^{\prime \prime}$ pixel $^{-1}$. To better subtract the background emission in the region of interest, we also got spectra of a blank region $\sim 0.5^{\circ}$ to the west of the target position. These 0.5 -hour spectra were in the same blue and red ranges as the target spectra.

Reference spectra of an Ar-Ne-He lamp were recorded before or after each observation to provide wavelength 


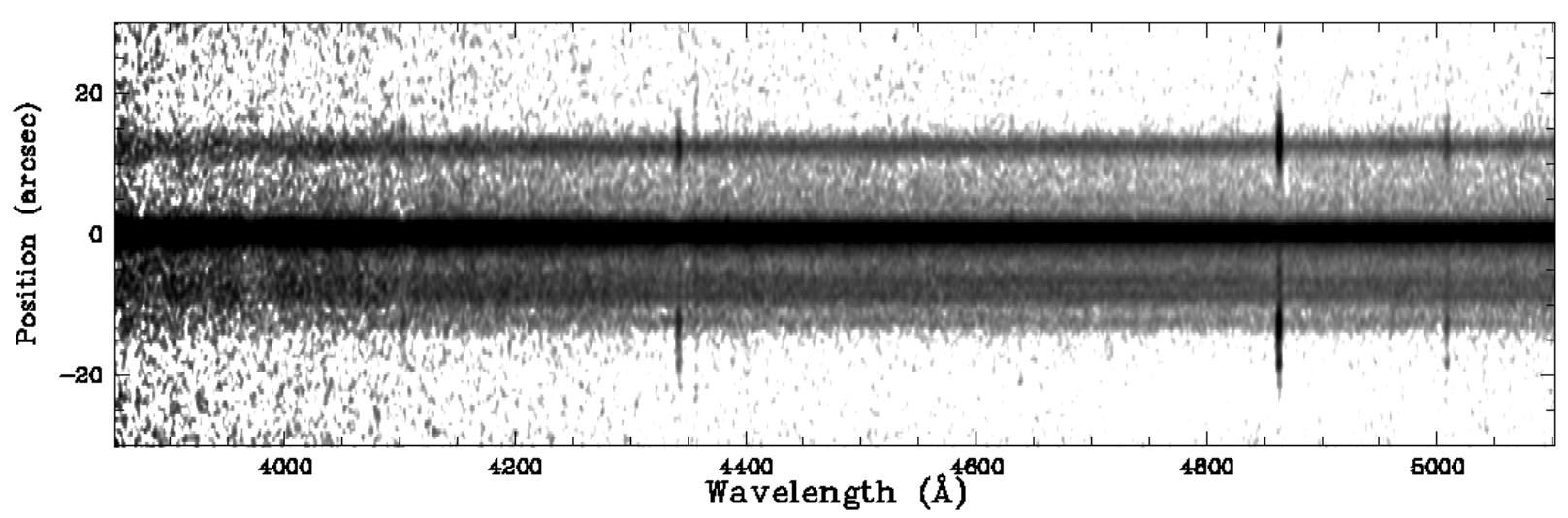

Fig. 2. The "blue" $2 \mathrm{D}$-spectrum for $\mathrm{PA}=-37^{\circ}$ corrected for CCD sensitivity. The frame is an average of three frames with an exposure time of 0.5 hour for each. The spectrum contains $\mathrm{H} \gamma \lambda 4340 \AA, \mathrm{H} \beta$, and [O III] $\lambda 5007 \AA$ emission lines, together with Balmer absorptions. The SSC is at the ordinate $Y=0^{\prime \prime}$. The NW direction corresponds to the negative ordinate. Two weaker, almost overlapping clusters inside the complex are seen at the ordinate $Y=-5^{\prime \prime}$ to $-10^{\prime \prime}$. They are clear in the H $\alpha$ profile in Fig. 5. There is weak blue emission inside the complex in contrast to the outside region at the ordinates $Y<-15^{\prime \prime}$ and $Y>15^{\prime \prime}$.
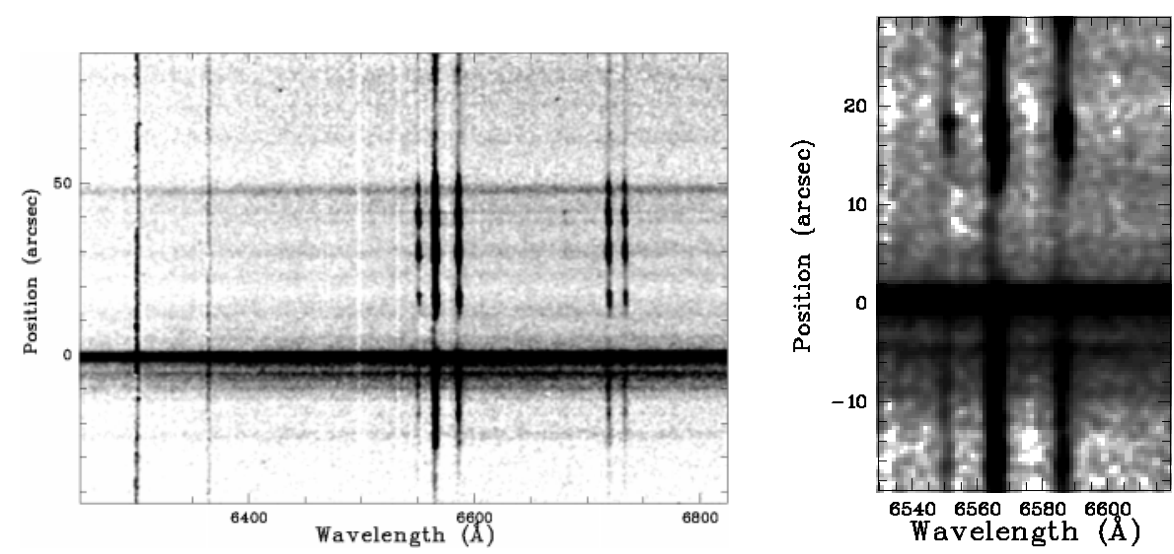

Fig. 3. Part of the "red" 2D-spectrum for $\mathrm{PA}=83^{\circ}$ which contains $\mathrm{H} \alpha(6562 \AA)$, [N II] $\lambda 6548,6584 \AA$ and [S II] $\lambda 6716,6730 \AA$ emission lines. The central star cluster is at the ordinate $Y=0^{\prime \prime}$. The direction to the West corresponds to negative ordinates. One more rather bright cluster inside the complex is at the ordinate $Y=-5^{\prime \prime}$. The arc-like form of $\mathrm{H} \alpha$ is seen for the ordinates between 0 and $+10^{\prime \prime}$ (enlargement on right). This corresponds to the high-velocity expansion with a negative displacement in Fig. 4. The same feature can be traced for the lines $[\mathrm{N} \mathrm{II}] \lambda 6584 \AA$ and $[\mathrm{S} \mathrm{II}] \lambda 6716 \AA$. These frames were made from an average of two frames with exposure times of 0.5 hour; each separate frame shows the arc-like feature of $\mathrm{H} \alpha$ near the ordinate $Y=5^{\prime \prime}$.

calibration. Spectrophotometric standard stars from Bohlin (1996) were observed for flux calibration. Observations have been conducted mainly under the software package NICE in MIDAS, described by Kniazev \& Shergin (1995). To be confident that we observed the same parts of the galaxy with the different set-ups on different nights, we employed the differential method of pointing the telescope, which was described in detail by Kniazev et al. (2001).

Procedures of primary data reduction included cosmicray removal in MIDAS $^{1}$, and bias subtraction and flat-field correction in IRAF. For the subsequent reduction of the long-slit spectra, we used IRAF ${ }^{2}$.

\footnotetext{
1 MIDAS is an acronym for the European Southern Observatory package - Munich Image Data Analysis System.

${ }^{2}$ IRAF: the Image Reduction and Analysis Facility is distributed by the National Optical Astronomy Observatories, which is operated by the Association of Universities for
}

After wavelength mapping and night sky subtraction using the blank field frames, each 2D frame was corrected for atmospheric extinction and flux calibrated. To derive the sensitivity curves, we used the spectral energy distributions of standard stars. Average sensitivity curves were produced for each observing night. An example of the results of such a reduction, part of a $2 \mathrm{D}$-spectrum, is shown in Fig. 3. To obtain the line-of-sight velocity distribution along the slit we used the methods described in Zasov et al. (2000).

\subsection{Keck-l telescope spectroscopy}

We supplemented our spectral analysis with highresolution echelle spectra from the Keck-I $10 \mathrm{~m}$ telescope,

Research in Astronomy, In. (AURA) under cooperative agreement with the National Science Foundation (NSF). 
Table 1. Journal of observations.

\begin{tabular}{|c|c|c|c|c|c|c|c|}
\hline $\begin{array}{l}\text { Date } \\
(1) \\
\end{array}$ & $\begin{array}{l}\mathrm{PA} \\
(2) \\
\end{array}$ & $\begin{array}{c}\text { Exposure } \\
\text { time }[\mathrm{s}] \\
(3) \\
\end{array}$ & $\begin{array}{c}\text { Wavelength } \\
\text { Range }[\AA] \\
(4) \\
\end{array}$ & $\begin{array}{c}\text { Dispersion } \\
{[\AA / \text { pixel }]} \\
(5) \\
\end{array}$ & $\begin{array}{l}\text { Slit } \\
{\left[{ }^{\prime \prime}\right]} \\
(6) \\
\end{array}$ & $\begin{array}{c}\text { Seeing } \\
{\left[{ }^{\prime \prime}\right]} \\
(7) \\
\end{array}$ & $\begin{array}{c}\text { Airmass } \\
(8) \\
\end{array}$ \\
\hline 26.07 .2000 & -37.3 & $1 \times 1800$ & $6015-7250$ & 1.2 & 2.0 & 1.4 & 1.11 \\
\hline 26.07 .2000 & -37.3 & $3 \times 1800$ & $3850-5100$ & 1.2 & 2.0 & 1.4 & 1.06 \\
\hline 26.07 .2000 & 83.3 & $2 \times 1800$ & $6015-7250$ & 1.2 & 2.0 & 1.4 & 1.11 \\
\hline 26.07 .2000 & 29.5 & $1 \times 1800$ & $6015-7250$ & 1.2 & 2.0 & 1.4 & 1.16 \\
\hline 28.07 .2000 & 83.3 & $3 \times 1800$ & $3850-5100$ & 1.2 & 2.0 & 2.2 & 1.06 \\
\hline 28.07 .2000 & 29.5 & $3 \times 1800$ & $3850-5100$ & 1.2 & 2.0 & 2.4 & 1.05 \\
\hline 28.07 .2000 & 29.5 & $2 \times 1800$ & $6015-7250$ & 1.2 & 2.0 & 2.7 & 1.12 \\
\hline
\end{tabular}

obtained for an independent project (Larsen et al. 2001) using the HIRES spectograph (Vogt et al. 1994). All details of the observations are described in that paper. For the present analysis, we use only one of the echelle orders, containing $\mathrm{H} \alpha$ emission with a full extent of $14^{\prime \prime}$ along a slit with a position angle of $-10^{\circ}$. Wavelength calibration was performed at SAO, based on the two night-sky lines seen in this order, namely $\mathrm{H} \alpha$ and $\mathrm{OH}$ $\lambda 6568.779 \AA$ (e.g., Osterbrock et al. 1996). This gives a scale of $0.048 \AA \mathrm{pixel}^{-1}$, or $2.2 \mathrm{~km} \mathrm{~s}^{-1}$ pixel $^{-1}$ at $\mathrm{H} \alpha$.

\section{Observational results}

\subsection{The velocity distribution from the 6-m telescope data}

The spectra show $\mathrm{H} \alpha$ emission over the whole extent of the field, $\sim 110^{\prime \prime}-130^{\prime \prime}$ long (depending on the PA of the slit), which is 3.2 to $3.8 \mathrm{kpc}$ for a distance to NGC 6946 of $6.0 \mathrm{Mpc}$ (Sharina et al. 1997). Figure 4 shows the $\mathrm{H} \alpha$ intensity and velocity measured with the $83^{\circ}$ slit, Fig. 5 shows the intensity and velocity from the $-37^{\circ}$ and $29^{\circ}$ slits, and Fig. 6 shows all three slits at higher spatial resolution centered on the SSC. The most detailed picture of the $\mathrm{H} \alpha$ velocity is seen in the spectrum with a slit orientation of $\mathrm{PA}=83^{\circ}$, which was obtained on the 1st night of observations with a seeing of $1.4^{\prime \prime}$. The $-37^{\circ}$ slit had the same seeing, and the $29^{\circ}$ slit had poorer seeing, $2.7^{\prime \prime}$ (see Table 1). All of these position-velocity diagrams are arranged in the same way: the left sides correspond to the W, SW or NW edges of the region, respectively (this is opposite to the orientation of the sky field in Fig. 1). The vertical lines are the position of the SSC. The background radial velocity of NGC 6946 is $125 \mathrm{~km} \mathrm{~s}^{-1}$ (Bonnarel et al. 1986,1988$)$ in the region near the SSC. This velocity is denoted by a dotted line in Figs. 4 and 5 .

The $\mathrm{H} \alpha$ velocity is disturbed by $\pm 10 \mathrm{~km} \mathrm{~s}^{-1}$ at several places where there are enhancements in the $\mathrm{H} \alpha$ emission. For example, the velocity has positive and negative excursions at each of the $\mathrm{H} \alpha$ intensity peaks at positions $30^{\prime \prime}$, $40^{\prime \prime}$, and $50^{\prime \prime}$ in Fig. 4. These disturbances could be unresolved shells caused by winds and old SN explosions (for a review of the bubble/shell phenomenon, see Tenorio-Tagle \& Bodenheimer 1988). At a distance of $40^{\prime \prime}$ in Fig. 4, both sides of what appears to be an ionized shell in the top panel are also visible in the middle panel at plus and minus velocities around the average. The negative velocity side of this shell, lying to the right in the figure, is brighter than the positive velocity side. Asymmetric $\mathrm{H} \alpha$ shells were also found by Martin (1996, 1998, and see the discussion of the "circumcluster" shell below). At the spectral resolution of the $6 \mathrm{~m}$ telescope, most small shells are unresolved and we see only the weighted average velocity in each slit position.

There is another positive velocity excursion at the distance of $10^{\prime \prime}$ in the $29^{\circ}$ slit. This corresponds to a faint filament seen in the $\mathrm{H} \alpha$-image in Fig. 1.

All three slits show a large, gradual, positive velocity perturbation around the systemic speed of $125 \mathrm{~km} \mathrm{~s}^{-1}$ for this region. It extends from about $-10^{\prime \prime}$ to $20^{\prime \prime}$ in all the slits, and has an amplitude of 40 to $50 \mathrm{~km} \mathrm{~s}^{-1}$. This feature is not a coherent shell since a receding part is not seen. It could be a systematic velocity pattern from the galaxy rotation curve and spiral arm flow, although the complex is at the end of a short arm, not inside a main arm (see the whole galaxy image in Elmegreen et al. 2000a).

In the central part of the $83^{\circ}$ slit there is a $-120 \mathrm{~km} \mathrm{~s}^{-1}$ swing in the ionized gas velocity from $170 \mathrm{~km} \mathrm{~s}^{-1}$ to $50 \mathrm{~km} \mathrm{~s}^{-1}$. We denote this feature as the "fast" expansion. The center of the fast expansion is displaced to the east of the SSC by $\sim 7^{\prime \prime}$, or $\sim 200 \mathrm{pc}$, and the extent of it is $\sim 9^{\prime \prime}$, or 260 pc (the tic marks on the $83^{\circ}$ slit in Fig. 1 indicate this extent). The center is near the position of a cluster that has an age of $\sim 30$ Myr, labeled " 33 " in Fig. 4 of Elmegreen et al. (2000a) and \#502 in Fig. 1 of Larsen et al. (2002); this cluster is seen in Fig. 1 here, slightly above the $83^{\circ}$ line, midway between the ticmarks). This cluster has an absolute visual magnitude of $M_{V}=-8.65$.

The fast expansion is also present in the $\mathrm{PA}=29^{\circ}$ slit (Fig. 5), where the amplitude of the negative motion is slightly smaller, $-80 \mathrm{~km} \mathrm{~s}^{-1}$, and the full extent of the region is larger, from $4^{\prime \prime}$ to $21^{\prime \prime}$ along the slit. The transverse distance between the $83^{\circ}$ and the $29^{\circ}$ slits at this position is comparable to the size of the disturbance in each slit, so the center of the fast expansion is somewhere between the two position angles, east of the SSC.

The $\mathrm{H} \alpha$ intensities and velocities in the central parts of the three slits are shown on an expanded scale in Fig. 6. 


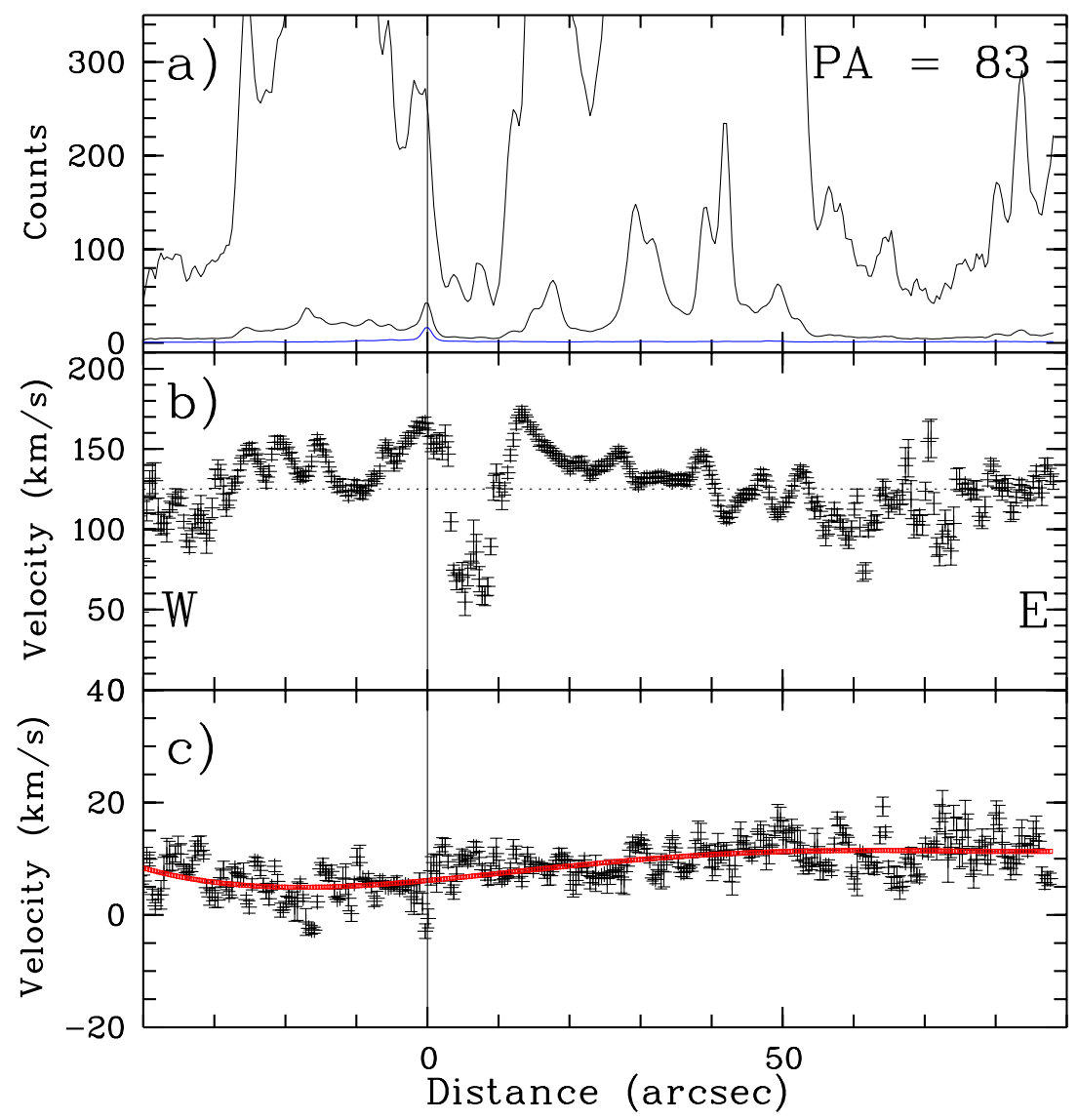

Fig. 4. $\mathrm{H} \alpha$ intensity and radial velocity distributions along the slit with $\mathrm{PA}=83^{\circ}$ in a region $130^{\prime \prime}(3.8 \mathrm{kpc})$ in extent. The vertical line at $X=0$ corresponds to the position of the SSC, which is $4^{\prime \prime}$ SSE of the center of the complex. The leftward direction in this plot corresponds to a rightward direction in Fig. 1 and in the sky. Upper panel: The lines at the bottom and middle show on a reduced scale (1/26 of the scale shown on $Y$-axis) the general behavior of the continuum intensity near H $\alpha$ and the flux of the $\mathrm{H} \alpha$-line, respectively. The upper line shows the profile of $\mathrm{H} \alpha$ flux along the slit with the continuum subtracted in real counts. Middle panel: The velocity profile of the $\mathrm{H} \alpha$ line, corrected using the night-sky line [O I] $\lambda 6363 \AA$. The horizontal line shows the background velocity of NGC 6946 near the complex as outlined by H $\alpha$ data from Bonnarel et al. (1986, 1988). Bottom panel: The deviations from the zero-position after wavelength transformation of the night-sky line [O I] $\lambda 6363 \AA$, with a fourth-order polynomial fit superimposed. This fitting curve was used to correct the first pass velocity curve and the result of the correction is shown in the middle panel $\mathbf{b}$ ). The rms of the distribution relative to the fitted curve is $\sim 3 \mathrm{~km} \mathrm{~s}^{-1}$.

The fast expansion down to a radial velocity of $50 \mathrm{~km} \mathrm{~s}^{-1}$ is clearly seen in the $83^{\circ}$ slit at the top of the figure, and partially seen in the $29^{\circ}$ slit in the middle panel. Other agitated emission occurs at the position of the SSC (the vertical line) in the $-37^{\circ}$ slit. This is the only $6-\mathrm{m}$ telescope slit that shows peculiar motion at the position of the SSC; the $-10^{\circ}$ slit used for the Keck data shows it better, as discussed in the next subsection.

The velocity-position profiles for the $83^{\circ}$ and $29^{\circ}$ slits in Fig. 6 show a broad range of velocities in the center of the fast emission. The $-37^{\circ}$ slit shows the same broad range in the center of the emission near the SSC. These dispersions in the centroid velocities of the emission lines correspond to broadenings in the actual line widths, suggesting that there are either multiple components or heightened turbulence. The velocity resolution of the instrument corresponds to a $F W H M$ of $\sim 145 \mathrm{~km} \mathrm{~s}^{-1}$ (3.2 $\AA$ ), based on the nearby reference spectrum and on night sky lines. Outside the complex, the linewidths are indistinguishable from the instrumental resolution. Within the complex, broadening over the instrumental profile varies between $10 \%$ and $20 \%$, and reaches a maximum of $\sim 25 \%$ near the SSC. These broadenings correspond to Gaussian velocity dispersions of $\sim 46 \mathrm{~km} \mathrm{~s}^{-1}$ near the SSC, and between 30 and $40 \mathrm{~km} \mathrm{~s}^{-1}$ in other parts of the complex out to $10-15^{\prime \prime}$ from the SSC, depending on direction. The high dispersion near the SSC is consistent with the Keck telescope data, which show an expanding shell there, as shown next.

\subsection{The velocity distribution from the Keck telescope data}

The 2D high resolution Keck-I echelle spectrum has greater sensitivity and better seeing $\left(0.9^{\prime \prime}\right)$ than the other spectra, so it is useful for faint small features. We only observed near the SSC, however, so many of the small velocity irregularities that occur in the 6 -m telescope slits 


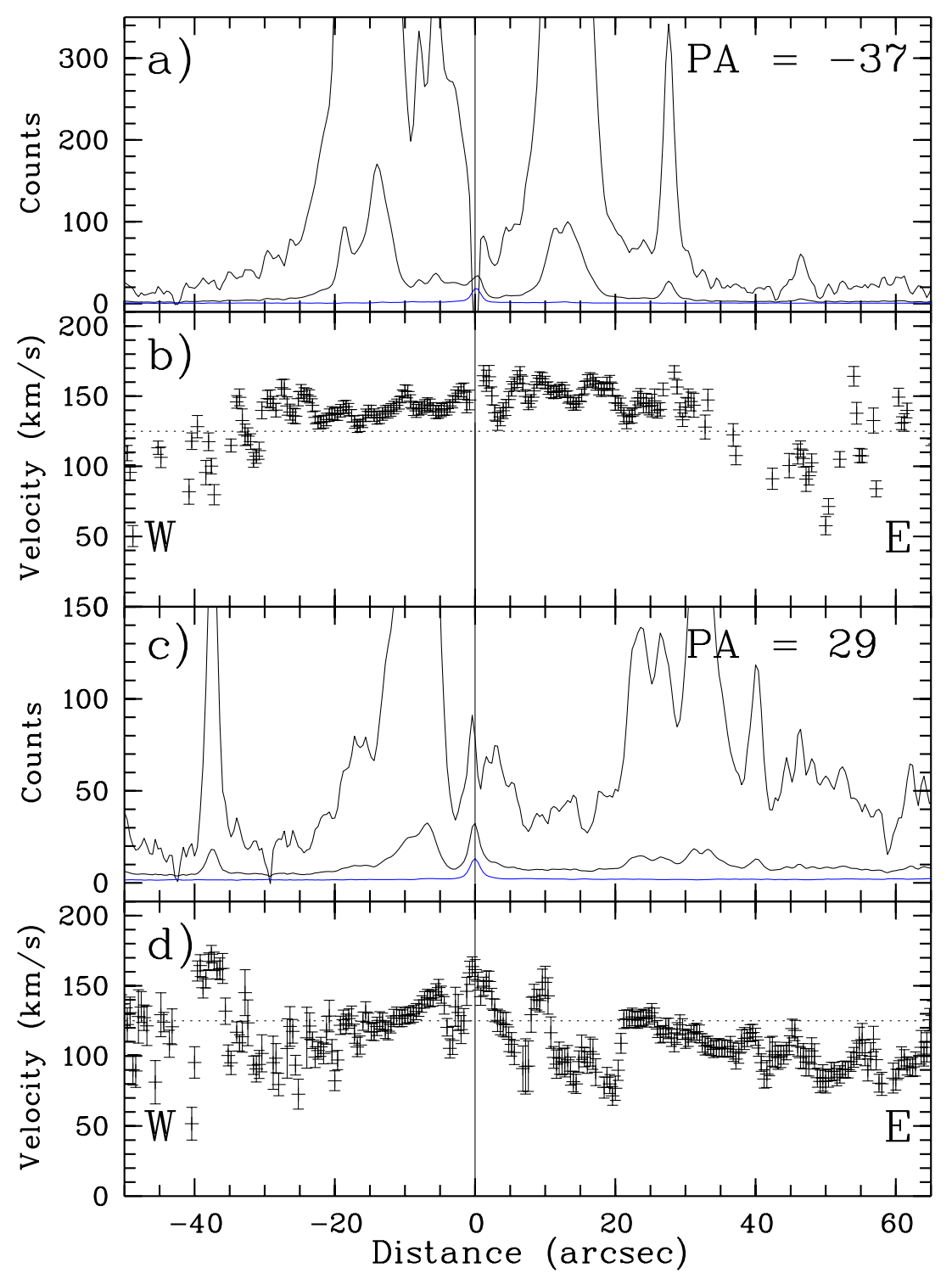

Fig. 5. The $\mathrm{H} \alpha$ intensity and radial velocity distributions along the slits with $\mathrm{PA}=-37^{\circ}$ and $\mathrm{PA}=29^{\circ}$, extending for $115^{\prime \prime}$ $(3.3 \mathrm{kpc})$. All panels are similar to those in Fig. 4.

cannot be seen with the Keck spectrum, nor can the largescale irregularities such as the fast expansion, which is comparable in size to the Keck slit $\left(14^{\prime \prime}\right)$.

The Keck spectrum in Fig. 7 shows another peculiar velocity feature in the Keck data. The velocity amplitude is $\sim 60 \mathrm{~km} \mathrm{~s}^{-1}$ and the diameter is $\sim 12^{\prime \prime}$, comparable to the fast feature seen in Fig. 4. The position angle of this slit is $-10^{\circ}$, which is similar to that of the $-37^{\circ}$ slit taken with the 6-m telescope.

The emission in the Keck spectrum has both positive and negative velocity components and extends on both sides of the SSC. It has all the characteristics of a fragmented shell with a radius of $6^{\prime \prime}(=170 \mathrm{pc})$ expanding at $\sim 60 \mathrm{~km} \mathrm{~s}^{-1}$. In what follows, we call it the "circumcluster shell". It is rather inhomogeneous, with slightly brighter emission located in the NNW sector (top of figure). In the SSE sector we see two separate knots near the relative velocity $V=0 \mathrm{~km} \mathrm{~s}^{-1}$. The innermost knot may be a dense ionized cloud inside the shell. The high-velocity tail of lower intensity in the SSE sector of the position-velocity diagram, stretching outward with relative velocities down to $-100 \mathrm{~km} \mathrm{~s}^{-1}$, could be a piece of the fast expansion seen in the other slits.

The $\sim 6^{\prime \prime}$ radius of the circumcluster shell is comparable to the distance between the SSC and the two dark clouds in the north (cf. Fig. 1). These clouds have an arched or shell-like appearance centered on the SSC, suggesting that the $\mathrm{H} \alpha$ circumcluster shell is the same as the partial dust shell.

\section{3. $\mathrm{H} \beta$ emission}

The $\mathrm{H} \beta$ emission inside the complex is in general significantly fainter than the $\mathrm{H} \alpha$. Within the radii where the fast expansion is seen in $\mathrm{H} \alpha$, no $\mathrm{H} \beta$ emission is detected for all three positions of the slit. This is not unreasonable 

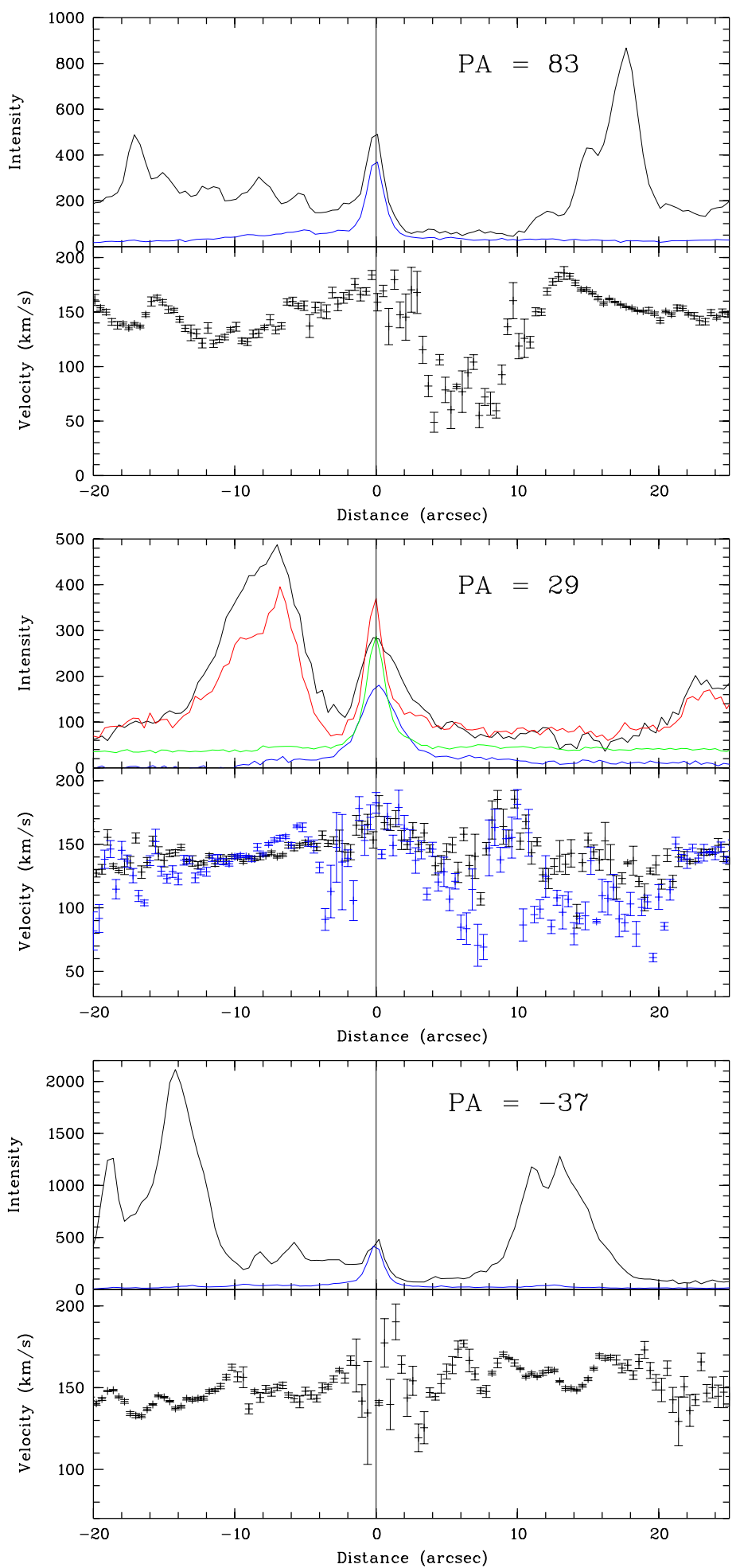

Fig. 6. Upper panel of each figure: the brightness profile of the $\mathrm{H} \alpha$ line + continuum (upper curve) and the brightness profile of the continuum near the $\mathrm{H} \alpha$ position (lower curve). Lower panel of each figure: the velocity profile of the H $\alpha$ line. The left hand parts of these three plots correspond respectively to W, SW and NW directions (see for reference Fig. 1). For PA $=29^{\circ}$, data are shown for both the first (sharper curves near position of SSC) and second nights. The significant effect of poor seeing is seen for the second night.

considering the noise. The Balmer decrement from extinction also decreases $\mathrm{H} \beta$ relative to $\mathrm{H} \alpha$. The extinction is at least partly caused by the Milky Way's extinction at the position of NGC $6946\left(l^{\mathrm{II}}=95.7^{\circ}, b^{\mathrm{II}}=11.7^{\circ}\right)$, which is
$A_{B} \sim 1^{\mathrm{m}} 5$ according to NED. This implies a difference in the obscuration between $\mathrm{H} \alpha$ and $\mathrm{H} \beta$ of $\sim 0{ }^{\mathrm{m}} 4$, or a factor of $\sim 1.5$. The $\mathrm{H} \alpha / \mathrm{H} \beta$ ratio then changes from the standard value of 2.88 for $T_{\mathrm{e}}=10000 \mathrm{~K}$ without extinction to 4.3 


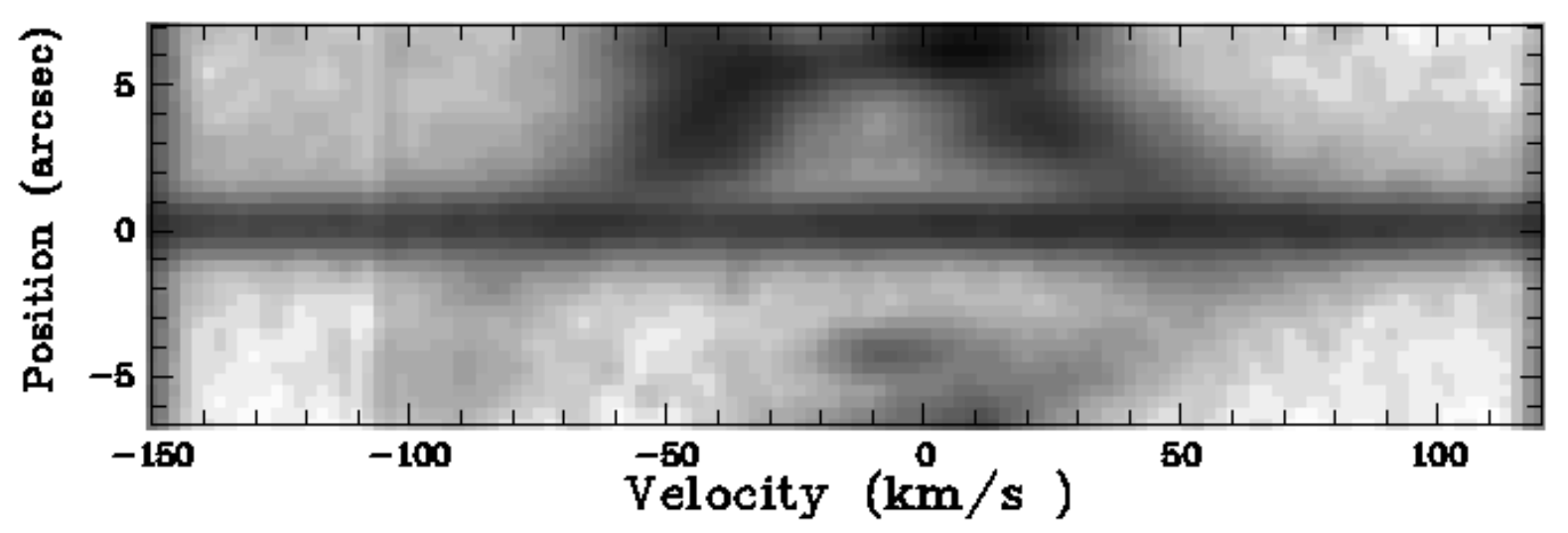

Fig. 7. A fragment of the Keck-I 2D echelle spectrum of the $\mathrm{SSC}$ and its vicinity near $\mathrm{H} \alpha$ with the $14^{\prime \prime}$ long slit at $\mathrm{PA}=-10^{\circ}$. The SSC is at the position along the slit corresponding to the ordinate $Y=0^{\prime \prime}$. Positive values of ordinate correspond to the NNW direction. The $X$-axis corresponds to the radial velocity of $\mathrm{H} \alpha$ emission relative to the SSC velocity, taken to be $150 \mathrm{~km} \mathrm{~s}^{-1}$ from the SSC spectrum. A shell with a radius of $R \sim 6^{\prime \prime}$ and a velocity of $60 \mathrm{~km} \mathrm{~s}^{-1}$ is centered on the SSC $\left(V_{\text {kin.center }}=144 \mathrm{~km} \mathrm{~s}^{-1}\right)$. A lower intensity feature with a radial velocity down to $-100 \mathrm{~km} \mathrm{~s}^{-1}$ relative to the SSC stretches to SSE. This is probably the fast expansion, seen in the middle panel of Fig. 4.

with extinction. The velocity field in $\mathrm{H} \beta$ is more noisy in the W, NW and SW directions; the positive wave in the velocity curve is well traced only in the $\mathrm{E}, \mathrm{NE}$, and $\mathrm{SE}$ sides.

\subsection{Physical conditions in the complex}

Determination of the physical conditions inside the star formation complex is difficult because of the intricate structure of ionized gas and the many sources of excitation and shocks. Little emission is seen in the [O III] lines $\lambda \lambda 4959,5007 \AA$, and there is no detectable emission in [O III] $\lambda 4363 \AA$.

In Fig. 8 we show the profiles of the line intensity ratios along the slit for $\mathrm{PA}=83^{\circ}$. The general behaviour of these profiles can be summarized as follows. In the close environment of the complex, the ratios are more or less typical of high-metallicity, low-excitation $\mathrm{H}$ II regions excited by massive stars. Over a larger region and especially inside the fast expansion, there is some increase of line ratios, probably indicating a contribution from shock excitation. The clear enhancement of line intensity ratios on both $\mathrm{E}$ and $\mathrm{W}$ peripheries of the complex, in the regions of very low $\mathrm{H} \alpha$ emission (with emission measure $E M \sim 15-30 \mathrm{pc} \mathrm{cm}^{-6}$ ), could be a Warm Ionized Medium (WIM) (see, e.g., Mathis 2000), a diffuse ionized gas (DIG), and/or a SNR (e.g., Galarza et al. 1999). Observations of WIM/DIG in the Milky Way and M 31 display line intensity ratios similar to those shown in the western, low-EM region (which are on the left in Fig. 8). However, the higher ratios seen in the eastern, low-EM regions are more compatible with those observed for SNRs (Galarza et al. 1999).

\subsection{Spectra of the SSC}

The spectrum of the SSC is well exposed in both red and blue in a total of 5.5 hours. Integrated over 7 pixels along the slit, it has a signal-to-noise ratio $(S / N) \sim 60$ at $\lambda 4800 \AA$ and $\sim 30$ near $\lambda 4000 \AA$ (see Fig. 11). The Balmer series in absorption from $\mathrm{H} \beta$ to $\mathrm{H}_{8}$ is well seen. Also several He I lines are seen in absorption at $\lambda \lambda 4388,4471,4921$ and $5015 \AA$. The radial velocity of the SSC determined from these (and $\lambda 6678$ of He I in red) absorption lines is $150 \pm 10 \mathrm{~km} \mathrm{~s}^{-1}$, which is close to the $\mathrm{H} \alpha$ velocity of the ionized gas in the position of the SSC.

The equivalent widths $(E W \mathrm{~s})$ of the Balmer absorption lines in blue may be used to estimate the age of the SSC (González Delgado et al. 1999). The observed EWs are given in Table 2 . The measurements were made according to the prescriptions given in González Delgado et al. in order to minimize systematic differences from their calibration. Typical $E W$ uncertainties are $\sim 10 \%$. Model results from González Delgado et al. are given on the right in the table. They assumed instantaneous star formation with a Salpeter IMF having a mass range from $M_{\text {low }}=1 M_{\odot}$ to $M_{\text {up }}=80 M_{\odot}$.

Helium lines can also be used to obtain independent estimates of age, but the uncertainties are higher by a factor of two because of their lower $E W \mathrm{~s}$, and in the range of ages defined by the Balmer lines, the $E W$ s of He have a low sensitivity to age.

The SSC is associated with an emission-line region, so $\mathrm{H} \beta$ absorption from the cluster can be contaminated by nebular emission. We model the observed intensities of Balmer emission lines assuming Case "B" recombination at $T_{\mathrm{e}}=10000 \mathrm{~K}$ and a Milky Way extinction $A_{B}=1.5$ (Schlegel et al. 1998). With these data we derive an $E W$ of $\mathrm{H} \beta$ emission $\sim 1.4 \pm 0.3 \AA$ and a corrected value of $\mathrm{H} \beta$ absorption from the SSC of $E W(\mathrm{H} \beta)=4.4 \pm 0.5 \AA$. According to Table 2 , this value of $4.4 \AA$ corresponds to an age of either $6 \mathrm{Myr}$ or $12 \mathrm{Myr}$. The photometric age is 15 Myr (Larsen et al. 2001).

For models with continuous star formation, the measured Balmer $E W \mathrm{~s}$ are less consistent with each other and correspond to ages of $\sim 30-50 \mathrm{Myr}$. Such long time scales 


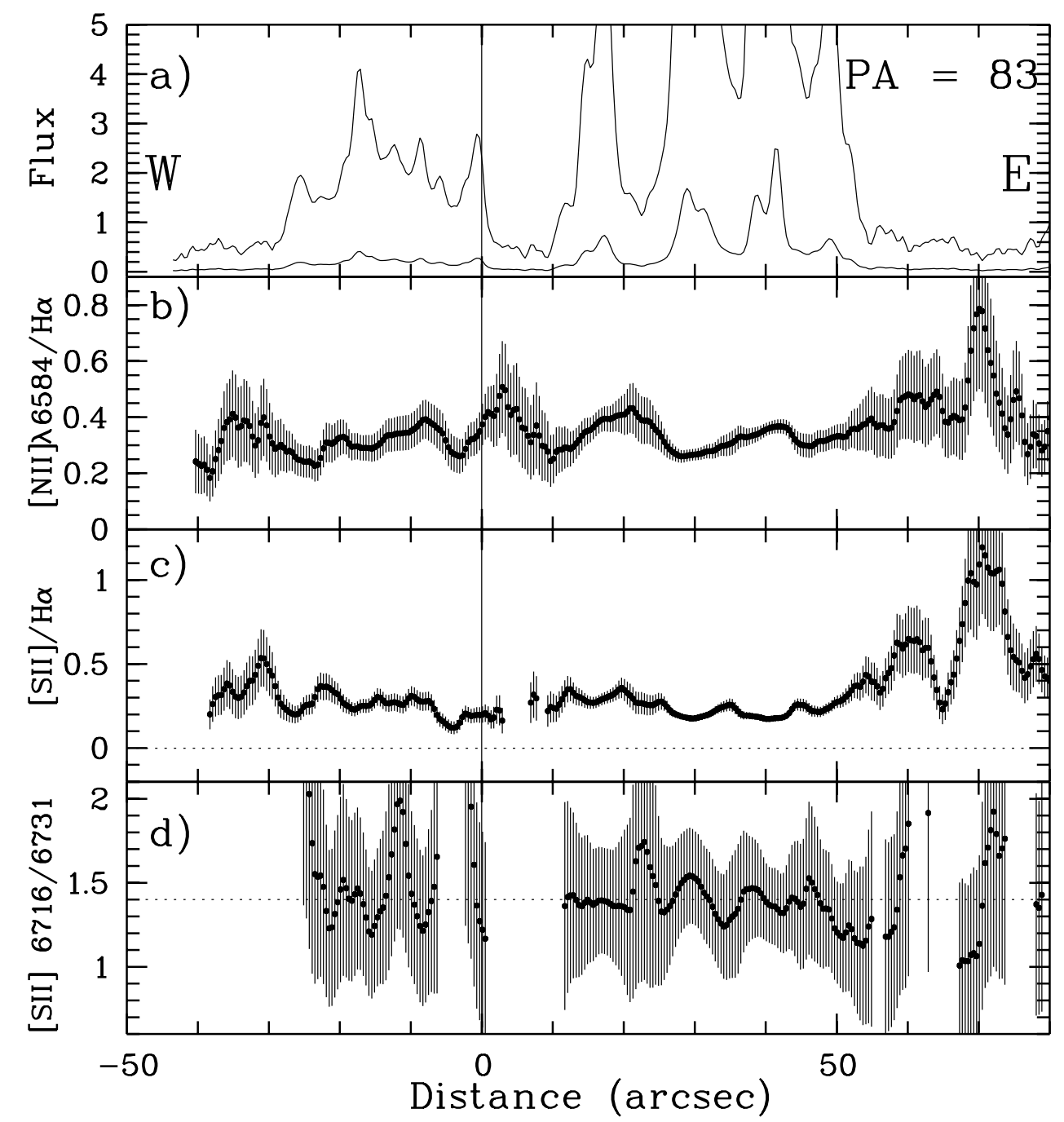

Fig. 8. Line intensity ratios along the slit for $\mathrm{PA}=83^{\circ}$. Top to bottom: a) Profile of $\mathrm{H} \alpha$ flux in units $10^{-16} \mathrm{erg}_{\mathrm{cm}}^{-2} \mathrm{~s}^{-1}$. Lower curve is scaled by a factor of $1 / 10$ to show bright regions. b) Profile of [N II6584]/H $\alpha$ ratio. While the background level of this parameter near the complex is $\sim 0.3$, it increases to $\sim 0.4$ in the region of slow perturbation, and rises up-to $\sim 0.5$ inside the fast expansion and up-to $0.6-0.8$ in the peripheral regions to the $\mathrm{E}$ and $\mathrm{W}$ with the low level of $\mathrm{H} \alpha$ emission. c) Profile of $[\mathrm{S} \mathrm{II}] 6716+6730 / \mathrm{H} \alpha$ ratio. Its background level of $\sim 0.3$ shows little change within the slow perturbation, however large variations are seen inside the fast expansion. The zero level of this ratio is shown for non-detected [S II] lines. This ratio is also enhanced up-to factors of 0.6-1.2 in peripheral regions with faint diffuse $\mathrm{H} \alpha$ emission. d) Profile of the density sensitive $[\mathrm{S} \mathrm{II}] 6716 /[\mathrm{S} \mathrm{II}] 6730 R_{[\mathrm{S} \text { II }]}$ ratio. For this ratio $\geq 1.5$ (dot-dash line) $n_{\mathrm{e}}<10 \mathrm{~cm}^{-3}$. For $R_{[\mathrm{S} \mathrm{II}]}=1.4,1.3,1.2,1.1,1.0$ and 0.9 respectively, $n_{\mathrm{e}}=40,130,240,380,580$ and $850 \mathrm{~cm}^{-3}$. The typical uncertainty in this ratio varies between 0.1 for the brightest features, as illustrated in Fig. 3, and 0.4-0.5 for the lowest brightness regions. Inside the fast expansion, these lines are almost everywhere undetectable, and the ratio is set to zero conditionally.

should be excluded because then the $E W$ of $\mathrm{H} \beta$ emission is expected to be 30 times higher than allowed by our data (Leitherer et al. 1999).

\subsection{Spectra of other star clusters within the complex}

The continuum spectra of most other clusters are too faint to measure $E W \mathrm{~s}$ of their Balmer absorption lines. One of the best spectra is of a faint object $\sim 5^{\prime \prime}$ to the west of the $\mathrm{SSC}$ at $\mathrm{PA}=83^{\circ}$ (seen toward negative positions in Fig. 3). This is the second object to the west of the SSC on the NOT $I$-band image (marked $16 \mathrm{Myr}$ in Fig. 4 of Elmegreen et al. (2000a). Because of poor seeing for this spectrum $\left(\sim 2.5^{\prime \prime}\right)$, it is highly probable that the brighter object (marked "19" in that previous figure) $\sim 1.5$ " to the north of object " 16 " gives a large contribution to the registered spectrum. Its energy distribution is somewhat bluer than that of SSC.

The Balmer lines $\mathrm{H} \gamma, \mathrm{H} \delta$ and $\mathrm{H}_{8}$ of this nearby cluster in the $83^{\circ}$ slit are well seen in absorption. Their $E W \mathrm{~s}$, uncorrected for the underlying gas emission lines, are in Table 2 in the column denoted by the $83^{\circ}$ position angle. Besides the $\mathrm{H} \beta$ line, which is heavily contaminated by emission, the $E W \mathrm{~s}$ of the other Balmer and He I lines are consistent within the uncertainties with those for the SSC, which suggests that this fainter cluster has an age 


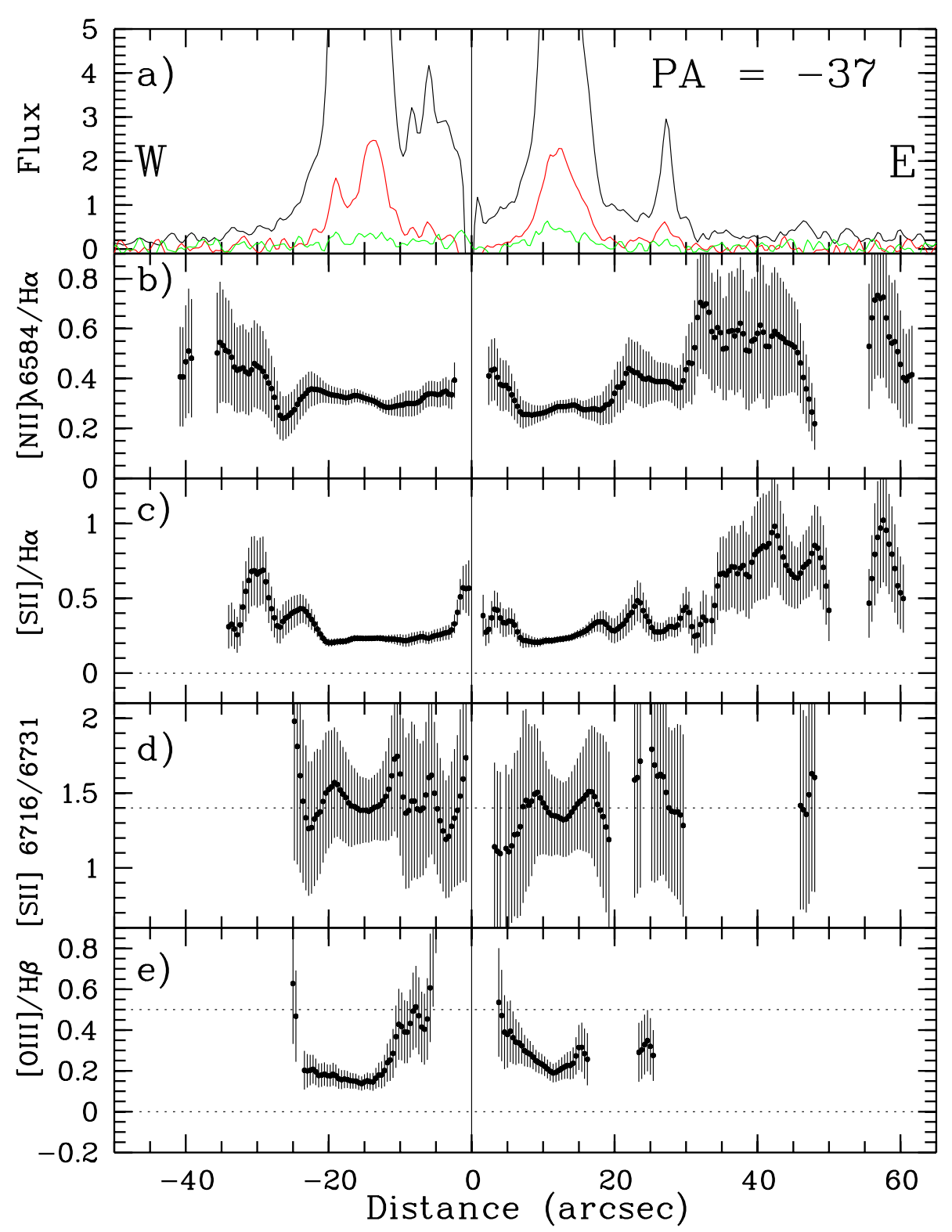

Fig. 9. Line intensity ratios along the slit for $\mathrm{PA}=-37^{\circ}$. a) Distribution along the slit of the line flux (in units $10^{-16} \mathrm{erg} \mathrm{cm}^{-2} \mathrm{~s}^{-1}$ ) for $\mathrm{H} \alpha$. The distribution of $\mathrm{H} \beta$ is shown by the middle line, and that of [O III] $\lambda 5007 \AA$ by the bottom line. Other panels show line intensity ratios as in the previous figure. The profile of the [O III] $\lambda 5007 / \mathrm{H} \beta$ ratio is shown in panel e).

that is about the same. However, its bluer spectral energy distribution in UV implies that it may be a bit younger, with an age of 6 to 9 Myr.

For the spectra of a fainter blend of two clusters in the $\mathrm{PA}=-37^{\circ}$ slit, the contamination by underlying emission in $\mathrm{H} \beta$ is even larger. However, the $E W \mathrm{~s}$ of absorption $\mathrm{H} \delta$ and $\mathrm{H}_{8}$ are practically non-affected by nebular emission. The values in Table 2 are consistent with an age less than 14 Myr.

The $E W$ of $\mathrm{H} \beta$ emission from the nebula can also be used as an estimator of the nearby cluster age. The $E W$ of $\mathrm{H} \beta$ from the vicinity of the the two faint star clusters in the $83^{\circ}$ slit is 6 to $6.5 \AA$. For an instantaneous starburst with Salpeter IMF between $M_{\text {low }}=1 M_{\odot}$ and
$M_{\text {up }}=100 M_{\odot}$ and solar metallicity, models in Leitherer et al. (1999) give an age of $7 \mathrm{Myr}$. For the faint pair of clusters seen in the spectrum with $\mathrm{PA}=-37^{\circ}, \mathrm{H} \beta$ emission is even stronger: $E W=8$ to $8.5 \AA$, which corresponds to an age of $6.3 \mathrm{Myr}$. These data indicate that both nearby cluster groupings are younger than the bright globular cluster. Younger isolated stars are also seen in the HST data (Larsen et al. 2002).

\subsection{Spectra of star clusters at the boundaries of the complex}

The same parameters can be used to estimate the ages of faint star clusters that delineate the arcs at the borders 


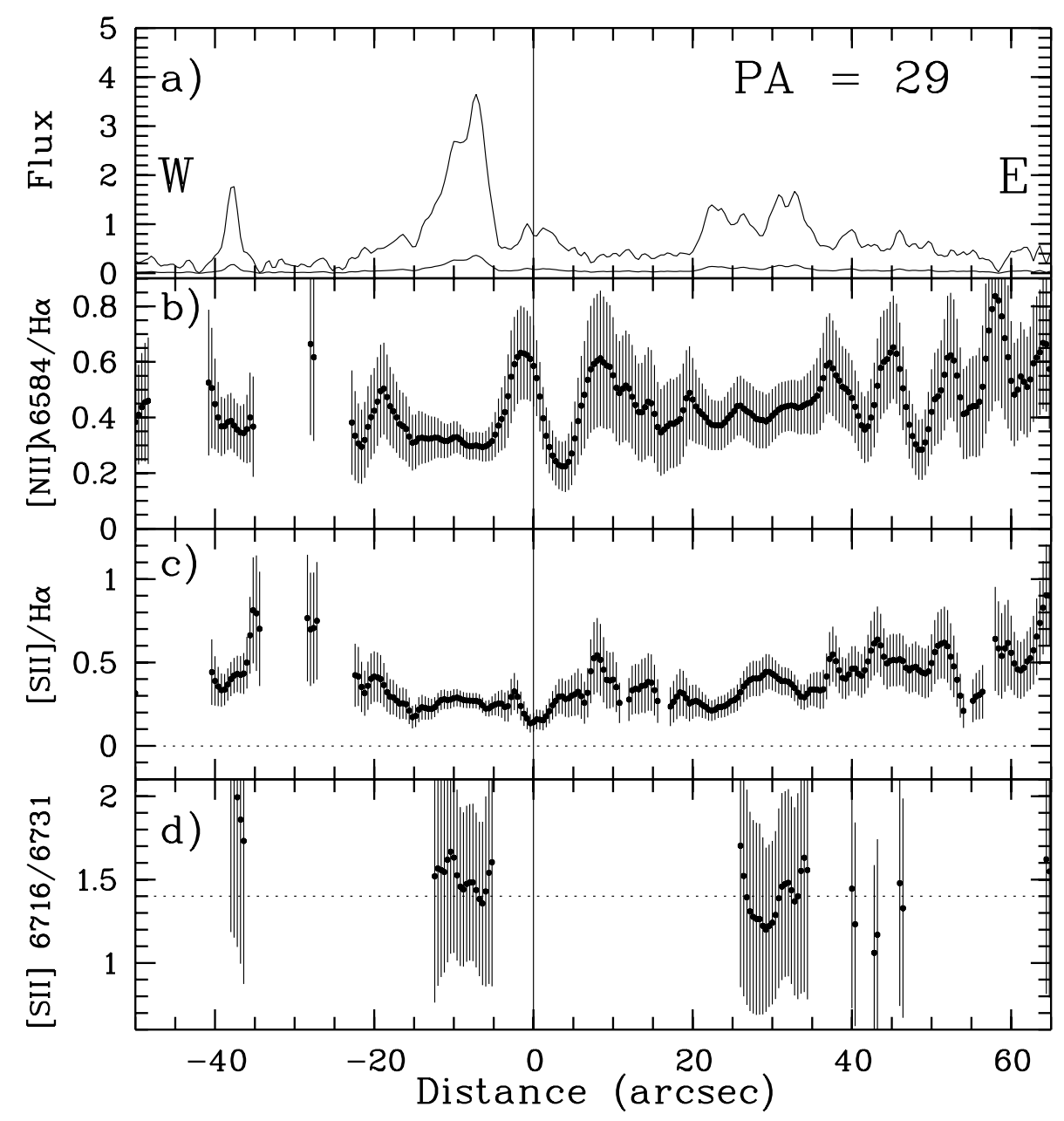

Fig. 10. Line intensity ratios along the slit for $\mathrm{PA}=29^{\circ}$. All panels are as in Fig. 8.

Table 2. Equivalent widths of absorption lines for the SSC and nearby clusters.

\begin{tabular}{|c|c|c|c|c|c|c|c|}
\hline $\begin{array}{l}(\AA) \\
(1)\end{array}$ & $\begin{array}{c}E W(\AA) \\
(2)\end{array}$ & $\begin{array}{c}\text { Nearby } \\
\text { clusters } \\
\mathrm{PA}=83^{\circ} \\
E W(\AA) \\
(3)\end{array}$ & $\begin{array}{r}\text { Nearby Blend } \\
\text { of } 2 \text { clusters } \\
\text { PA }=-37^{\circ} \\
E W(\AA) \\
(4)\end{array}$ & $\begin{array}{l}\text { Model } \\
6 \mathrm{Myr} \\
E W(\AA) \\
\quad(5)\end{array}$ & $\begin{array}{l}\text { Model } \\
8 \mathrm{Myr} \\
E W(\AA) \\
\quad(6)\end{array}$ & $\begin{array}{l}\text { Model } \\
12 \mathrm{Myr} \\
E W(\AA) \\
\quad(7)\end{array}$ & $\begin{array}{c}\text { Model } \\
14 \mathrm{Myr} \\
E W(\AA) \\
(8)\end{array}$ \\
\hline $4861 \mathrm{H} \beta$ & $3.0 \pm 0.3$ & $-1.9 \pm 0.3$ & $-3.9 \pm 0.6$ & 4.6 & 4.0 & 4.6 & 5.8 \\
\hline $4340 \mathrm{H} \gamma$ & $5.8 \pm 0.6$ & $4.7 \pm 0.4$ & $3.9 \pm 0.8$ & 3.8 & 4.3 & 4.5 & 5.6 \\
\hline $4101 \mathrm{H} \delta$ & $6.4 \pm 0.6$ & $6.9 \pm 0.7$ & $5.6 \pm 1.0$ & 5.0 & 5.7 & 6.2 & 7.2 \\
\hline $3889 \mathrm{H}_{8}$ & $3.6 \pm 0.6$ & $2.9 \pm 0.7$ & $4.3 \pm 1.2$ & 3.8 & 4.6 & 4.8 & 5.6 \\
\hline $4922 \mathrm{He} \mathrm{I}$ & $0.8 \pm 0.2$ & $0.8 \pm 0.2$ & - & 0.90 & 0.52 & 0.49 & 0.92 \\
\hline $4471 \mathrm{He} \mathrm{I}$ & $0.9 \pm 0.2$ & $0.8 \pm 0.2$ & - & 0.87 & 0.80 & 0.94 & 1.1 \\
\hline
\end{tabular}

Model: instantaneous SF at solar metallicity from González Delgado et al. (1999)

Minus in $E W$ s of $\mathrm{H} \beta$ means that this is the value for emission.

of the complex. Their continuum is too faint to measure their Balmer absorption lines, but well enough exposed to get an estimate of the $E W$ s of $\mathrm{H} \beta$ nebular emission, and thus to get some insight about their ages. As an example, we measured this parameter for the two most visible faint clusters on both sides of the SSC in the blue spectrum acquired with the slit at $\mathrm{PA}=-37^{\circ}$. In Fig. 2, the positions of these clusters are $+12^{\prime \prime}$ (SE cluster) and $-12^{\prime \prime}$
(NW cluster). The $E W$ s of their $\mathrm{H} \beta$ emissions, calculated from $1 \mathrm{D}$ spectra summed over 8 pixels along the slit $\left(3.2^{\prime \prime}\right.$, where the cluster continua are still visible) are, respectively, $22 \AA$ and $42 \AA$. After correction for the $E W$ of $\mathrm{H} \beta$ absorption, which is expected to be $\sim 5 \AA$ for an age between 5 and $13 \mathrm{Myr}$, we derive true $E W$ s of $\mathrm{H} \beta$ nebular emission of $27 \AA$ and $47 \AA$, respectively. From the models by Leitherer et al. (1999) with the same IMF as above, we 
derive ages for these star forming regions of 5.7 Myr for the SE cluster and 5.3 Myr for the NW cluster. We have checked these small ages also from independent data for $E W(\mathrm{H} \alpha)$, obtained from the red spectrum. Their $E W \mathrm{~s}$ of 160 and $270 \AA$ give ages (Leitherer et al. 1999) consistent with those derived from the $E W(\mathrm{H} \beta)$. As seen in Fig. 5 the full extent of $\mathrm{H} \alpha$ around these clusters is a bit larger than the range over which the $1 \mathrm{D}$ spectrum was obtained. This leads to an underestimate of related $E W \mathrm{~s}$ of $\mathrm{H} \beta$ and $\mathrm{H} \alpha$ by 10 to $20 \%$, and to a decrease in the age estimates by $\sim 5$ to $10 \%$.

Well-exposed spectra of these two clusters in red also show strong lines of $[\mathrm{N}$ II $] \lambda \lambda 6548,6584 \AA$. Their fluxes relative to $\mathrm{H} \alpha$ can be used to get some empirical estimate of metallicities in the $\mathrm{H}$ II regions. The relation:

$12+\log (\mathrm{O} / \mathrm{H})=1.02 \times \log (I([\mathrm{~N} \mathrm{II}]) / I(\mathrm{H} \alpha))+9.36$

was given by van Zee et al. (1998). We get for these two clusters from our data $12+\log (\mathrm{O} / \mathrm{H})=8.97$ and 8.93, respectively, with formal uncertainties of 0.2 dex. This oxygen abundance corresponds to the solar value (see, e.g., Anders \& Grevesse 1989).

\section{Discussion}

We have undertaken a spectroscopic study of the velocity field of ionized gas within and near a peculiar star complex in the SW part of the giant spiral galaxy NGC 6946 . Long-slit spectra taken at three position angles separated by $\sim 60^{\circ}$ sample the main features of the velocity field. The spectra of the young central star cluster and several fainter clusters were also obtained with sufficiently high $S / N$ ratio to measure the equivalent widths of their Balmer absorption lines and/or the $E W$ s of $\mathrm{H} \beta$ emission from the surrounding Hil regions. This allows us to get information about their ages and motions relative to that of the ionized gas.

The following parameters related to the processes taking place in this stellar complex can be derived from the ionized gas kinematics.

\subsection{Characteristic timescales for the peculiar motions}

We estimate the characteristic timescales of the three large velocity features connected with the complex. Two of them are seen best on the position-velocity diagram in $\mathrm{H} \alpha$ for the slit position with $\mathrm{PA}=83^{\circ}$. The diameter of the slow perturbation with a positive wave in the velocity curve and an amplitude of $\sim 50 \mathrm{~km} \mathrm{~s}^{-1}$ (relative to the background velocity field) is $\sim 25^{\prime \prime}$ or $\sim 730$ pc. If we use the formula $v=0.6 R / t$ from Weaver et al. (1977) for the speed of a shell due to the pressure of a hot superbubble, then the age of this feature would be $0.6 R / v=4.4 \mathrm{Myr}$. For the Sedov (energy-conserving) phase of a fast shell with an instantaneous energy injection, the age would be $t=$ $0.4 R / v=2.9 \mathrm{Myr}$. For a spiral-arm flow, the characteristic time scale would be $2 R / v=15 \mathrm{Myr}$.

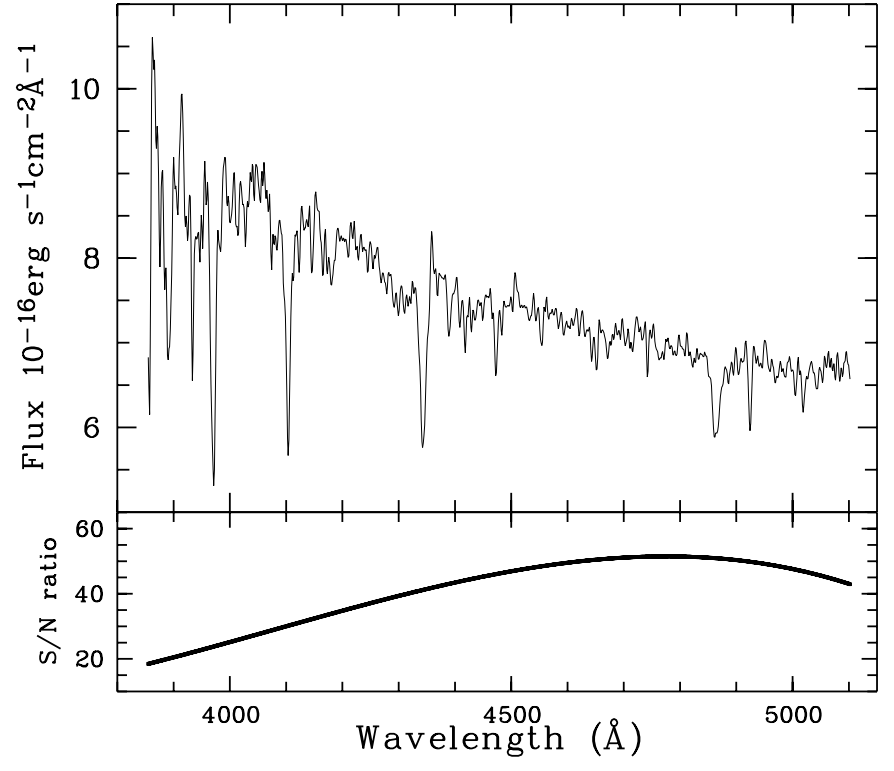

Fig. 11. 1D composite spectrum of the SSC, obtained by averaging of $1 \mathrm{D}$ spectra for the slits with $\mathrm{PA}=29^{\circ}$ and $\mathrm{PA}=83^{\circ}$. The equivalent widths of the Balmer and He I absorption lines are consistent with an age of the SSC equal to $\sim 12 \mathrm{Myr}$ (in the approximation of instantaneous star formation). The signal-tonoise ratio of the composite spectrum as a function of wavelength is shown in the bottom panel.

The negative velocity feature $7^{\prime \prime}$ east of the SSC (the fast expansion) has a smaller age. Its diameter in the $83^{\circ}$ slit is $9^{\prime \prime}$, or $260 \mathrm{pc}$, and its current speed is $120 \mathrm{~km} \mathrm{~s}^{-1}$ (this is the amplitude of the negative motion relative to the level of the slow perturbation near the SSC). Applying the same formulae for winds and supernovae, we get an age between 0.4 and 0.6 Myr. The circumcluster shell has a diameter of $\sim 12^{\prime \prime}$ or $350 \mathrm{pc}$, and a speed of $60 \mathrm{~km} \mathrm{~s}^{-1}$ giving it an age of $\sim 1.5 \mathrm{Myr}$.

The fast expansion and circumcluster shell are younger than the SSC and its neighbouring faint clusters. Older regions with velocities of $\leq 20 \mathrm{~km} \mathrm{~s}^{-1}$ would be more difficult to detect in such a disturbed region. Many of the small velocity perturbations in the spectra could be older.

\subsection{Kinetic energy of the perturbed regions}

The sizes and velocities of the expansion regions give their kinetic energies, $E=0.5 M v^{2}$ for mass $M$ and peculiar velocity $v$. The mass of the fast expansion can be estimated from the $\mathrm{H} \alpha$ luminosity in the high velocity part of the emission line. The $\mathrm{H} \alpha$ surface brightness in the line is $\sim 6 \times 10^{-17} \mathrm{erg} \mathrm{cm}^{-2} \mathrm{~s}^{-1}$ (square arcsec) ${ }^{-1}$. This corresponds to an emission measure of $\left\langle n_{\mathrm{e}}^{2}\right\rangle d=30 \mathrm{pc} \mathrm{cm}^{-6}$ for electron density $n_{\mathrm{e}}$ and line of sight depth $d$ in the shell (Marlowe et al. 1995; Osterbrock 1989). The depth is unknown but is likely to be less than $1 / 12$ the radius $R=130 \mathrm{pc}$ of the expanding region, or $d<11 \mathrm{pc}$. This is because the ratio of the shocked density to the ambient density is $R / 3 d$ for a spherical shell, and this ratio exceeds 4 for a radiative shock. Then, $n_{\mathrm{e}}>1.7 \mathrm{~cm}^{-3}$ and 

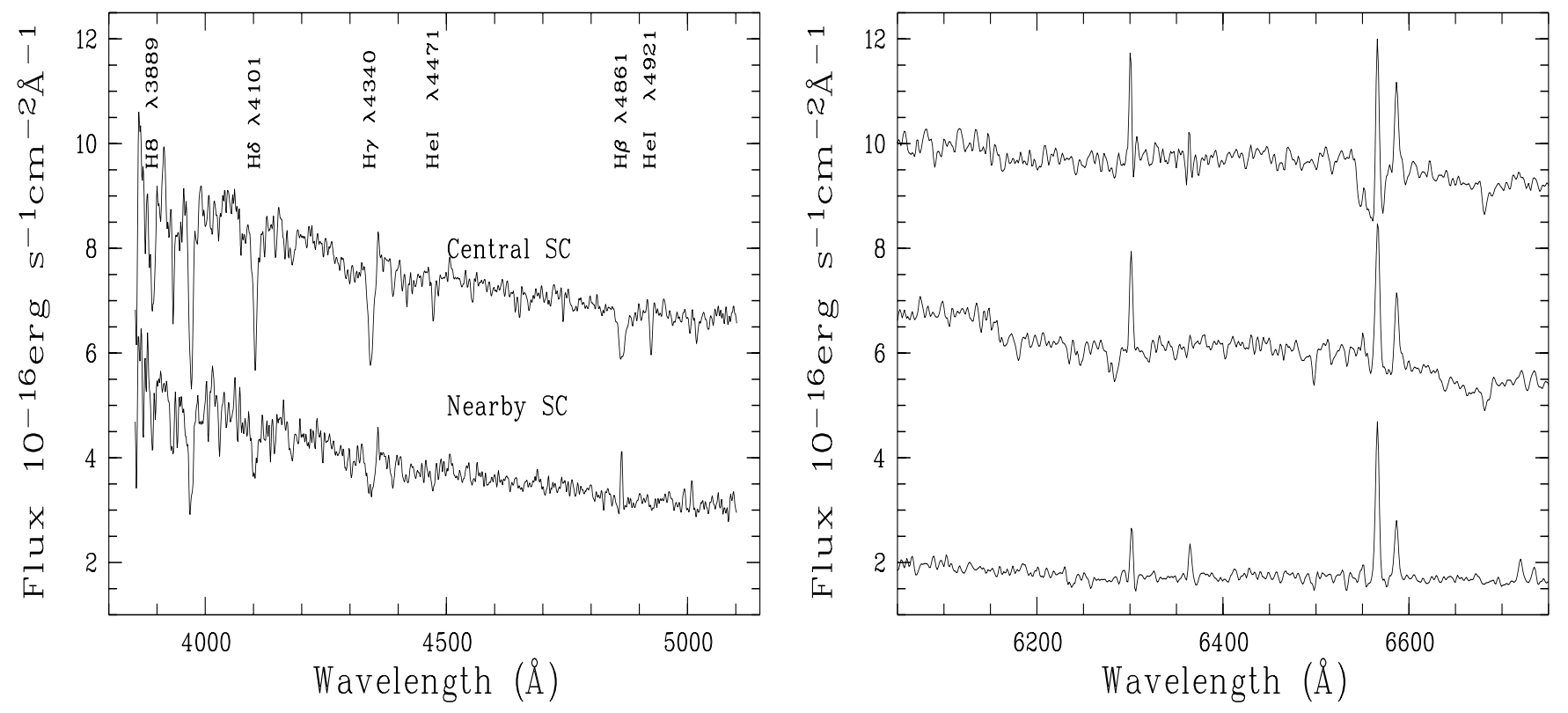

Fig. 12. Left panel: blue spectra of the SSC (top) and nearby star cluster (bottom) at PA $=83^{\circ}$. The latter shows a steeper rise of its spectrum toward the UV in comparison to that of SSC. The Balmer series is well seen in absorption, as well as the He I $\lambda 4471,4921 \AA$ lines. Right panel: red spectra of the SSC with PA $=-37^{\circ}$ on the top and PA $=83^{\circ}$ in the middle; the spectrum of the nearby star cluster is on the bottom. Emission from the surrounding ionized gas is superposed on the absorption spectra of the star clusters. He I $\lambda 6678 \AA$ absorption in the spectrum of SSC is seen. Redward of [O I] $\lambda 6300 \AA$ and $\lambda 6364 \AA$ non-perfect sky subtraction creates artificial emission. The red spectrum of the SSC observed with PA $=-37^{\circ}$ is shifted up by +3.5 units for convenience. The difference between the red spectrum of the SSC at $\mathrm{PA}=-37^{\circ}$ and that at $\mathrm{PA}=83^{\circ}$ is less than $5 \%$ in the range from 6000 to $6800 \AA$ but it jumps up to $\sim 20 \%$ in the small region around H $\alpha$, which is seen in absorption. However the fluxes of emission $\mathrm{H} \alpha$ line in these spectra differ by only $\sim 6 \%$.

a spherical half-shell (we observe only the approaching side) would have a mass of $2 \pi R^{2} d n_{\mathrm{e}} \mu<6 \times 10^{4} M_{\odot}$ for mean atomic weight including helium and heavy elements, $\mu=2.2 \times 10^{-24} \mathrm{gm}$. The corresponding kinetic energy is $<9 \times 10^{51}$ ergs.

The initial energy of an explosion is greater than the mechanical energy because of adiabatic and radiative losses during the expansion. The initial energy of a supernova is $E \sim 5.3 \times 10^{43} n_{0}^{1.12} v^{1.40} R^{3.12}$ erg for preshock density $n_{0}$ in $\mathrm{cm}^{-3}, v$ in $\mathrm{km} \mathrm{s}^{-1}$, and $R$ in pc (Chevalier 1974). If $n_{0}<0.4 \mathrm{~cm}^{-3}$, which is consistent with the shell density limit and emission measure given above, then $E<6 \times 10^{52} \mathrm{ergs}$. If the expanding region is only a halfshell, then $E<3 \times 10^{52} \mathrm{ergs}$. Similarly, the injected energy for a wind-driven shell in the snow-plow phase exceeds the mechanical energy by a factor of $\sim 5.1$ (Weaver et al. 1977), making $E<4.6 \times 10^{52}$ ergs. The upper limits to these energies arise from the uncertainty in shell thickness, given that the only observation of mass is from an emission measure and this depends on the square of the density. The actual energy is probably close to these values because magnetic fields should dominate the pressure in the swept-up gas, so the shell density will be close to the minimum estimate. In any case, the energy limit is equivalent to several tens of supernovae detonating within about a half million years.

The energy of the circumcluster shell is $0.5 M v^{2}=2.4 \times$ $10^{52} n_{0}$ erg for $v=60 \mathrm{~km} \mathrm{~s}^{-1}$ and $M=(4 \pi / 3) R^{3} n_{0} m_{\mathrm{H}}$ in a spherical shell with radius $R=170 \mathrm{pc}$ and pre-shock density $n_{0}$. If $n_{0} \sim 0.1$ atom $\mathrm{cm}^{-1}$ as above, then the kinetic energy is $2 \times 10^{51} \mathrm{ergs}$, and the injected energy is $\sim 5$ times larger for wind power. These numbers imply a total energy equivalent to $10 \mathrm{SNe}$ released in the last $\sim 1.5 \mathrm{Myr}$.

The SSC has enough stars to power both the circumcluster shell and the fast expansion. Figure 13 shows the wind and supernova mechanical luminosities from a $10^{6} M_{\odot}$ cluster like the SSC over a period of $15 \mathrm{Myr}$, which is about the SSC age. A Salpeter IMF from 1 to $100 M_{\odot}$ is assumed, using the data in Starburst99 (Leitherer et al. 1999, scale down all luminosities by a factor of 2.5 for this assumed cluster mass if the lower limit to the IMF is $0.1 M_{\odot}$ ). The wind power (plotted as a dotted line) comes mostly during the first $6 \mathrm{Myr}$, while the supernovae power (solid line) starts after $3.5 \mathrm{Myr}$. Between 9.5 Myr and 12.5 Myr there is a large burst of supernova power. This latter interval corresponds to the expansion time of the circumcluster shell. The integrated supernova energy during this time in the figure is $10^{55} \mathrm{ergs}$, which is 5000 times the circumcluster shell energy. The SSC energy from the last $0.5 \mathrm{Myr}$ is $\sim 2 \times 10^{53}$ ergs if we use the low power part of Fig. 13 that lies to the right of the peak. This is $\sim 20$ times the mechanical energy in the fast expansion, which has about this age. Evidently there is more than enough power coming from the SSC to drive the expansions observed spectroscopically. 


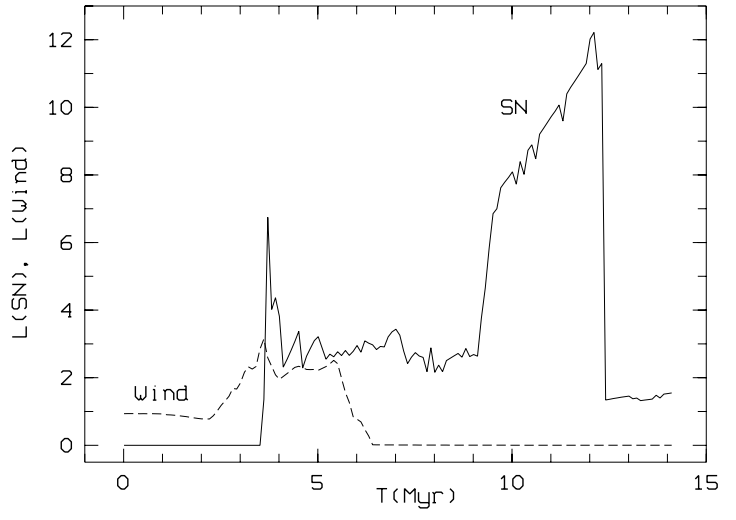

Fig. 13. The history of the mechanical luminosity of a $10^{6} M_{\odot}$ cluster in units of $10^{40} \mathrm{erg} \mathrm{s}^{-1}$. The dotted line is the wind luminosity and the solid line is for supernova.

A large part of the SSC energy may have blown out of the galaxy. The dust clouds in the north and northwest and the high density gas behind the circular arc in the west (Elmegreen et al. 2000a) seem to have blocked the expansion in this direction, leaving the winds and supernova energy relatively free to move eastward. This is where the lowest $\mathrm{H} \alpha$ emission measure is, indicating a cleared cavity, and also where the large negative velocities are found. The lack of $\mathrm{H} \alpha$ at the rest velocity suggests that the whole galactic disk in this region is being pushed toward us at $\sim 100 \mathrm{~km} \mathrm{~s}^{-1}$. That would imply either that the SSC is displaced slightly toward the backside of the disk and has ionized and accelerated the whole disk, or that the backside part of an ionized shell has already blown so far out of the disk, away from the Lyman continuum source, that its $\mathrm{H} \alpha$ emission is now weak.

There is no reason to postulate any special energy source for the high speed expansion other than supernovae from the SSC. Some of the pre-supernova stars may have evaporated from the SSC and moved to the east, aiding in the expansion there, or a more exotic type of star or binary system could have moved there, exploding as a hypernova (Paczynski 1998; Turatto et al. 2000). The high energy and short time scale of this expansion, $\sim 10^{52}$ ergs in $\sim 0.5 \mathrm{Myr}$, and the lack of any significant clusters in the center of the fast region, suggests that a lot of SSC energy got there quickly, as in the ejected hypernova scenario. Hot gas could have moved there quickly too.

The possibility of a hypernova ejection from the SSC follows from recent results for the dynamical evolution of star clusters. Massive stars sink to a cluster core rapidly and dynamical interactions lead to their ejection. Kroupa (2000) showed that during the first 10 to $50 \mathrm{Myr}$, between $10 \%$ and $50 \%$ of the most massive stars in a cluster migrate to distances larger than the tidal diameter. This explains the fact that most Cepheids (which are the most massive stars in a cluster) occur in the halos (Efremov 2000). It also explains the many massive stars found in the halo of the R136 cluster in the LMC (Selman et al. 1999; Brandle et al. 2001).

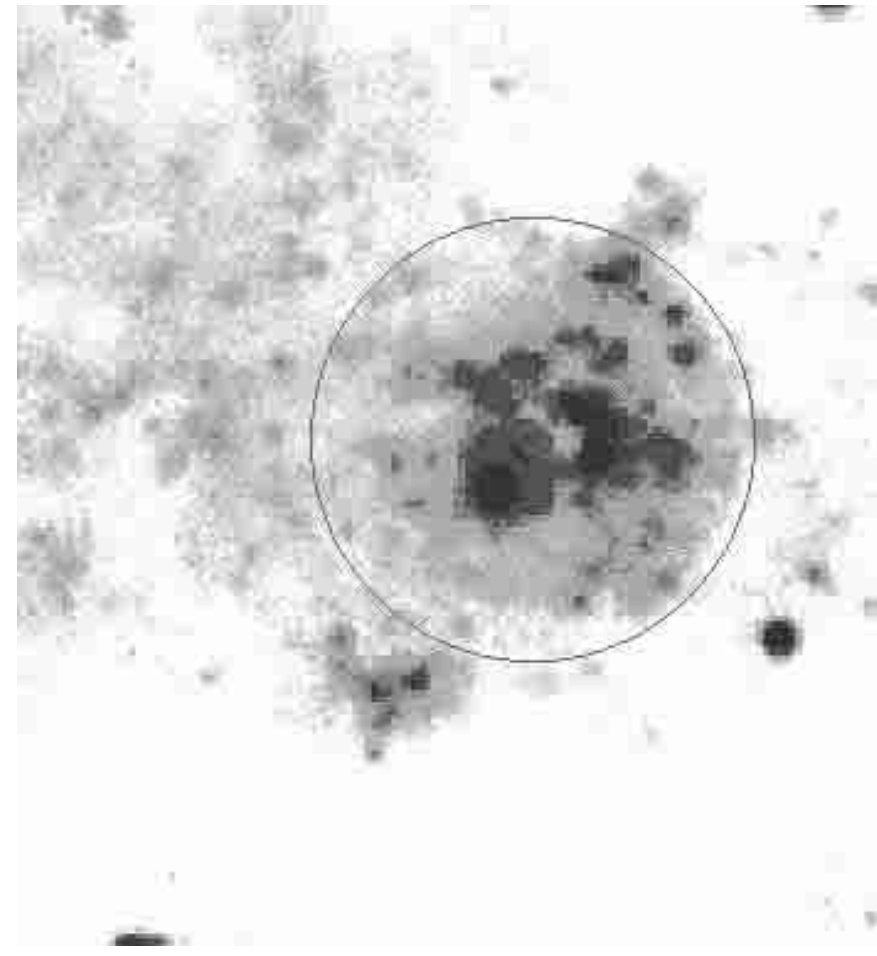

Fig. 14. The bubble complex from an enhanced version of the NOT color image. Note the perfect semiring for the Western rim. The center of the drawn circle is noted by the small grey circle.

For a normal IMF, the most massive stars in the SSC should have a mass of $\sim 100 M_{\odot}$ (Larsen et al. 2001), which is suitable for a hypernova. However, in the dense cores of clusters like this, merging events should occur which lead to the formation of supermassive and fast rotating stars or even black holes of a few hundred $M_{\odot}$ (Portegies Zwart et al. 1999; Matsushita et al. 2000; Ebisuzaki et al. 2001). These objects are the primary candidates for hypernovae outbursts that may be connected with GRB events (Paczynski 1998). The most massive stars of a $12 \mathrm{Myr}$ old cluster have normally much lower mass. The suggestion therefore follows that the object which might have formed the fast expansion in the hypernova scenario resulted from a recent merger and ejection in the SSC. The possibility of the formation and ejection of GRB progenitors in dense clusters was discussed in Efremov (2000).

\subsection{The relative velocities and positions of the perturbations and the SSC}

The radial velocity of the SSC is $\sim 150 \mathrm{~km} \mathrm{~s}^{-1}$. The kinematic center of the slow perturbation is $\sim 125 \mathrm{~km} \mathrm{~s}^{-1}$, and the kinematic center to the sides of the fast expansion is $\sim 170 \mathrm{~km} \mathrm{~s}^{-1}$. The circumcluster shell has the same kinematic center as the SSC $\left(\sim 150 \mathrm{~km} \mathrm{~s}^{-1}\right)$. The SSC and the fast expansion have central velocities that are all comparable to that of the surrounding gas. In Fig. 5 of Bonnarel et al. (1988), the region of the complex is within a large loop of isolines between 125 and $140 \mathrm{~km} \mathrm{~s}^{-1}$. The SSC and 
fast expansion velocities are only slightly larger than the maximum average for the region, and the slow perturbation is comparable to the minimum average. The velocity peculiarities of $\pm 20 \mathrm{~km} \mathrm{~s}^{-1}$ are not strong enough to suggest any particular scenario for the origin of the whole region. It lies near the end of an arm segment or spur, where density wave flow speeds are expected to be comparable to these velocity fluctuations.

The morphology and intensity of star formation in this region are unusual so there might be an unusual explanation for its origin. There is a comet-like shape to the whole region, with a sharp circular edge in the west and a more open, tail-like structure in the east. Figure 14 shows an enhanced version from the NOT image. This shape could have been triggered by the oblique impact of an extragalactic cloud, from east to west. The impact had to be oblique so that the resulting disk velocities were not too different from the local rest speed. Cloud-disk collisions have been suggested for NGC 6946, but HVCs and HI holes are only known in other regions (Boulanger \& Viallefond 1992; Kamphuis 1993). There is no prominent HI hole in the complex, only a small hole in the region of the fast expansion east of the $\mathrm{SSC}$, as seen in Fig. 1f of Frick et al. (2000). An oblique collision of a high-velocity cloud with a magnetized gaseous disk results in MHD waves, gas vortices, and Parker instabilities, but no prominent hole (Santillán et al. 1999). All of this, along with the resulting shock wave collisions, could explain some the peculiarities of the complex, including the discrete epochs of star formation, the dust lane across it, the circular Western rim, and the small perturbations in the HII velocities. The SSC could then be the result of the shock wave interaction. These possibilities are considered in more detail elsewhere (Efremov 2002).

A problem with the impact theory is that the comet also looks like a normal spiral arm spur with a continuum of blue stars inside, and there are other similar spurs in this galaxy. This makes the origin of the complex look more like a spiral arm process than a random cloud impact. Also, some of the asymmetric morphology could result from a high-pressure expansion toward the east using energy from the SSC and a density gradient toward the west (see the blister models in Icke 1981). There is in fact a higher density of HI to the west in the Frick et al. map, and there is more extinction there in our optical images as well (see Elmegreen et al. 2000a).

\section{Conclusions}

Long-slit spectroscopy of a unique stellar complex in NGC 6946 has shown several interesting features that shed light on this unusual object. We draw the following conclusions:

1. Position-velocity diagrams of $\mathrm{H} \alpha$ emission in the complex indicate the presence of three large-scale motions: a slow perturbation with a full size of 700-800 pc and a velocity of $\sim 50 \mathrm{~km} \mathrm{~s}^{-1}$, a fast expansion, with a size of $260 \mathrm{pc}$ and a velocity of $120 \mathrm{~km} \mathrm{~s}^{-1}$, and a circumcluster shell with a size of $350 \mathrm{pc}$ and a velocity of $60 \mathrm{~km} \mathrm{~s}^{-1}$. The latter is centered in both position and velocity on the super star cluster. The fast expansion may be centered 200 pc away from this cluster but still receive energy from it. The large energy and short time scale for the fast expansion suggests that an ejected hypernova might have been involved.

2. Well sampled spectra of the young globular cluster show Balmer and He I absorption lines with radial velocity of $\sim 150 \mathrm{~km} \mathrm{~s}^{-1}$, close to that of the ionized gas in this region. The $E W \mathrm{~s}$ of absorption lines and of the $\mathrm{H} \beta$ emission line are used to date the ages of the SSC and two nearby clusters. The age of the SSC is $12 \pm 2 \mathrm{Myr}$, and those of the nearby fainter clusters are significantly smaller, $\sim 7$ Myr. Some clusters on the periphery of the complex are even younger, with ages of $\sim 5.5 \mathrm{Myr}$. These ages are in good agreement with those found from the $U B V$ photometry obtained with the HST (Larsen et al. 2002). The HST data also obtained ages for other clusters and found no pattern with position.

3. The ratios of emission line intensities ([N II $] 6584 / \mathrm{H} \alpha$ and ([S II] $6716+6731) / \mathrm{H} \alpha$ ) indicate that shock excitation can contribute in the region of the fast expansion.

4. The overall velocities of the SSC and most of the $\mathrm{H}$ II gas have no strong deviations from the rotation curve of NGC 6946.

Acknowledgements. This research has made use of the NASA/IPAC Extragalactic Database which is operated by the Jet Propulsion Laboratory, California Institute of Technology, under contract with the NASA. The use of Digitized Sky Survey and NASA Astrophysics Data System is gratefully acknowledged. Yu.E. appreciates the support from the RFBR, grant 00-02-17804 and from the Conseil for the Support of the Scientific Schools, grant 00-15-96627. Yu.E. was also partially supported by Federal Purposive Scientific-Technical program, contract \#40.022.1.1.1101. B.G.E and S.L. were supported by HST grants GO-08715.02-A and GO-08715.05-A. EJA acknowledges partial support from DGICYT trough grants PB97-1438-C02-02 and by Research and Education Council of the Autonomous Government of Andalucia (Spain). Thanks are due to the referee M. Rieke, who made several useful suggestions which helped to improve the presentation of results and discussion.

\section{References}

Afanasiev, V. L., Burenkov, A. N., Vlasyuk, V. V., \& Drabek, S. V. 1995, SAO RAS, Internal Rep., No. 234

Anders, E., \& Grevesse, N. 1989, Geochim. Cosmochim. Acta, 53, 197

Bohlin, R. C. 1996, AJ, 111, 1743

Bonnarel, F., Boulesteix, J., \& Marcelin, M. 1986, A\&AS, 66, 149

Bonnarel, F., Boulesteix, J., Georgelin, Y. P., et al. 1988, A\&A, 189,59

Boulanger, F., \& Viallefond, F. 1992, A\&A, 266, 37 
Brandle, B., Chernoff, D. F., \& Moffat, A. F. J. 2001, in Extragalactic star clusters, ed. E. Grebel, D. Geisler, \& D. Minniti, Proc. IAU Symp., 207, in press

Chevalier, R. 1974, ApJ, 188, 501

Ebisuzaki, T., Makino, J., Tsuru, T. G., et al. 2001, ApJ, 562, 19

Efremov, Y. N. 1995, AJ, 110, 2757

Efremov, Y. N. 1999, Astron. Lett., 25, 100

Efremov, Y. N. 2000, Astron. Lett., 26, 558

Efremov, Y. N. 2001, Astron. Rep., 45, 769

Efremov, Y. N. 2002, Astron. Rep., 46 (10), in press

Elmegreen, B., Efremov, Y. N., \& Larsen, S. 2000a, ApJ, 535, 748

Elmegreen, B. G., Efremov, Y. N., Pudritz, R., \& Zinnecker, H. 2000b, in Protostars and Protoplanets IV, ed. V. Mannings, A. P. Boss, \& S. S. Russel (Univ. Arizona Press), 179

Frick, P., Beck, R., Shukurov, A., et al. 2000, MNRAS, 318, 925

Galarza, V. C., Walterbos, R. A. M., \& Braun, R. 1999, AJ, 118,2775

González Delgado, R. M., Leitherer, C., \& Heckman, T. M. 1999, ApJS, 125, 489

Hodge, P. 1967, PASP, 79, 29

Icke, V. 1981, ApJS, 45, 585

Kamphuis, J. J. 1993, Ph.D. Thesis, Univ. of Groningen

Kniazev, A. Y., \& Shergin, V. S. 1995, SAO RAS Internal Rep., No. 249, 1

Kniazev, A. Y., Pustilnik, S. A., Pramsky, A. G., \& Ugryumov, A. V. 2001, A\&A, 371, 404

Kroupa, P. 2000, in Massive stellar clusters, ed. A. Lanson, \& C. Boily (ASP Conf. Ser.), 233 [astro-ph/0001202]

Larsen, S., \& Richtler, T. 1999, A\&A, 345, 59

Larsen, S. S., Brodie, J., Elmegreen, B., et al. 2001, ApJ, 556, 801

Larsen, S. S., Efremov, Y. N., Elmegreen, B., et al. 2002, ApJ, 567,896

Leitherer, C., Schaerer, D., Goldader, J. D., et al. 1999, ApJS, 123,3
Marlowe, A. T., Heckman, T. M., Wyse, R. F. G., \& Schommer, R. 1995, ApJ, 438, 563

Martin, C. 1996, ApJ, 465, 680

Martin, C. 1998, ApJ, 506, 222

Mathis, J. S. 2000, ApJ, 544, 347

Matsushita, S., Kawabe, R., Matsumoto, H., et al. 2000, ApJ, 545, L107

Meaburn, J. 1980, MNRAS, 192, 365

Osterbrock, D. 1989, Astrophysics of Gaseous Nebulae and Active Galactic Nuclei (Mill Valley: University Science Books)

Osterbrock, D. E., Fulbright, J. P., Martel, A. R., et al. 1996, PASP, 108, 277

Paczynski, B. 1998, ApJ, 494, L45

Portegies Zwart, S. F., Makino, J., McMillan, S. L. W., \& Hut, P. 1999, A\&A, 348, 117

Santillán, A., Franco, J., Martos, M., \& Kim, J. 1999, ApJ, 515,657

Schlegel, D. J., Finkbeiner, D. P., \& Douglas, M. 1998, ApJ, 500,525

Selman, F., Melnick, J., Bosch, G., \& Terlevich, R. 1999, A\&A, 347,532

Sharina, M., Karachentsev, I. D., \& Tikhonov, N. A. 1997, Astronomy Lett., 23, 373

Tenorio-Tagle, G., \& Bodenheimer, P. 1988, ARA\&A, 26, 145

Turatto, M., Suzuki, T., Mazzali, P. A., et al. 2000, ApJ, 534, L57

van Zee, L., Salzer, J. J., Haynes, M. P., O’Donoghue, A. A., \& Balonek, T. J. 1998, AJ, 116, 2805

Vogt, S. S., Allen, S. L., Biglow, B. C., et al. 1994, Proc. SPIE, 2198, 362

Weaver, R., McCray, R., Castor, J., Shapiro, P., \& Moore, R. 1977, ApJ, 218, 377

Westerlund, B. E., \& Mathewson, D. S. 1966, MNRAS, 131, 371

Zasov, A., Kniazev, A., Pustilnik, S., et al. 2000, A\&AS, 144, 429 